\title{
"Unusual" critical states in type-II superconductors
}

\author{
E. H. Brandt ${ }^{1}$ and G. P. Mikitik ${ }^{1,2}$ \\ ${ }^{1}$ Max-Planck-Institut für Metallforschung, D-70506 Stuttgart, Germany \\ ${ }^{2}$ B. Verkin Institute for Low Temperature Physics 8 Engineering, \\ National Ukrainian Academy of Sciences, Kharkov 61103, Ukraine
}

(Dated: August 3, 2021)

\begin{abstract}
We give a theoretical description of the general critical states in which the critical currents in typeII superconductors are not perpendicular to the local magnetic induction. Such states frequently occur in real situations, e.g., when the sample shape is not sufficiently symmetric or the direction of the external magnetic field changes in some complex way. Our study is restricted to the states in which flux-line cutting does not occur. The properties of such general critical states can essentially differ from the well-known properties of the usual Bean critical states. To illustrate our approach, we analyze several examples. In particular, we consider the critical states in a slab placed in a uniform perpendicular magnetic field and to which two components of the in-plane magnetic field are then applied successively. We also analyze the critical states in a long thin strip placed in a perpendicular magnetic field which then is tilted towards the axis of the strip.
\end{abstract}

PACS numbers: 74.25.Sv, 74.25.Qt

\section{INTRODUCTION}

The concept of the critical state introduced by Charles Bean ${ }^{1}$ is widely used to describe various physical phenomena in the vortex phase of type-II superconductors, see, e.g., Refs. 2, 3 and citations therein. According to Bean, in the critical state of type-II superconductors with flux-line pinning, the driving force of the currents flowing in this state is balanced by the pinning force acting on the vortices. The critical state is characterized by the component of the current density flowing perpendicular to the flux lines, $j_{c \perp}$, since only this component generates a driving force. It is assumed in the critical-state theory that this $j_{c \perp}$ is known, i.e., it is a given function of the magnetic induction $\mathbf{B}, j_{c \perp}=j_{c \perp}(\mathbf{B})$, and the problem of this theory is to find the appropriate distribution of the magnetic fields and currents in the critical state. Below, for simplicity, we shall assume that the magnetic fields $\mathbf{H}$ in the superconductor considerably exceed the lower critical field $H_{c 1}$, and so we put $\mathbf{B}=\mu_{0} \mathbf{H}$ throughout the paper. Beside this, we deal only with bulk superconducting samples, assuming that all their dimensions noticeably exceed the London penetration depth, and we consider the critical state macroscopically, averaging vortex structures and the appropriate microscopic currents over a scale exceeding the intervortex spacing.

Hereafter we shall call the critical states "Bean critical states" if the current density $\mathbf{j}$ is perpendicular to the local magnetic field $\mathbf{H}$ at every point of a superconductor, $\mathbf{j}=\mathbf{j}_{\perp}$, and thus $j=j_{\perp}=j_{c \perp}$. This definition imposes limitations on the direction of the currents in the critical state, but it does not imply constancy of $j_{c \perp}$, e.g., $j_{c \perp}(H)$ can be as in the Kim model $\underline{\underline{4}}$ The Bean critical states can be found from the static Maxwell equations,

$$
\operatorname{rot} \mathbf{H}=\mathbf{j}, \quad \operatorname{div} \mathbf{H}=0,
$$

and the conditions on the current density

$$
\begin{gathered}
\operatorname{div} \mathbf{j}=0, \\
j_{\perp}=j_{c \perp}, \quad j_{\|}=0,
\end{gathered}
$$

where $j_{\|}$is the component of the current density along the local magnetic field $\mathbf{H}$. Such states usually occur when the shape of the superconductor is sufficiently symmetric and the external magnetic field $\mathbf{H}_{a}$ is applied along a symmetry axis, so that the direction of the currents is dictated by the symmetry of the problem. Most of the known solutions of the critical state problem describe just these Bean states. For example, this is the well-known solution for an infinite slab in an external magnetic field parallel to its surface, $\stackrel{1}{=}$ and also the solution for an infinitely long cylinder with arbitrary crosssection in a magnetic field parallel to its axis since the currents flow perpendicular to this axis. $\stackrel{2}{=}$ Bean critical states also occur in infinitely long and thin strips ${ }^{5.6 .7}$ and in thin disks 8 in a perpendicular magnetic field even if $j_{c \perp}$ depends on $|\mathbf{B}| \equiv B$ or on the angle between $\mathbf{B}$ and the normal to the sample plane. $\frac{9,10,11}{1 f}$ the applied magnetic field is tilted to the plane of an infinitely long strip $\frac{12,13,14,15}{1}$ or slab $\frac{16}{16}$ but remains perpendicular to the sample axis, the critical currents flow along this axis, and a Bean critical state occurs. Further examples of the Bean critical states in samples of a complex shape can be found in Ref. 17, 18, 19, 20,21. A characteristic feature of all these Bean critical states is that the perturbation of the current distribution caused by a change of the applied field propagates into the sample as a sharp front at which the direction of the currents changes abruptly.

In real samples of nonsymmetric shape, or when the applied magnetic field changes not only in amplitude but also in its direction, adjacent flux lines may be slightly rotated relative to each other in the critical state. This rotation generates a component of the current along the magnetic field, ${ }^{22} \mathbf{j}_{\|}$. The rotation of flux-lines can lead to their mutual cutting, 2,22 Flux line cutting occurs when 
the component of the current density parallel to the magnetic field, $j_{\|}$, exceeds some longitudinal critical current density $j_{c \|}$. In this situation a vortex ${ }^{23}$ or a vortex array ${ }^{24}$ becomes unstable with respect to a helical distortion, and the growth of this distortion leads to flux-line cutting. When both $j_{\|}$and $j_{c \perp}$ are equal to their critical values $j_{c \|}$ and $j_{c \perp}$, respectively, the so-called double critical state ${ }^{22,25}$ occurs in the superconductor $\stackrel{26}{2}$ For example, this state appears in some region of a superconducting sample $\frac{25,27}{5}$ when a rotating magnetic field of constant magnitude is applied to a superconducting disk (or slab) in its plane ${ }^{28,29,30}$ The double critical state can be still described by Eq. (11), (2), but with the following conditions on the current density $\mathbf{j}=\mathbf{j}_{\perp}+\mathbf{j}_{\|}$:

$$
j_{\perp}=j_{c \perp}, \quad j_{\|}=j_{c \|} .
$$

The concept of the critical state with flux-line cutting was further developed in Refs. 32,33 34 to explain the observed suppression of the magnetic moment of a superconducting slab under the action of an ac magnetic field $34,35,36$

However, in many real situations a change of the direction of the external magnetic field or a nonsymmetric shape of the sample does not lead to flux cutting in the superconductor, i.e., $j_{\|}$does not reach $j_{c \|}$ in the critical state. In such situations there is no explicit condition on the magnitude of $j_{\|}$except that $j_{\|}<j_{c \|}$, and the static equations (11) and (2) with the only restriction $j_{\perp}=j_{c \perp}$ are not sufficient to find the distributions of the magnetic field $\mathbf{H}(\mathbf{r})$ and current density $\mathbf{j}(\mathbf{r})$ in the critical state. This problem for the special case of a slab with an in-plane magnetic field was solved in Refs. 25,27. The full set of the critical-state equations for arbitrary shape of the sample and for any quasistatic evolution of the vector of the applied magnetic field $\mathbf{H}_{a}$ was obtained in Ref. 37, where it was also shown that in contrast to the common Bean critical states, a perturbation of the current distribution in such critical states propagates into the sample smoothly in a diffusive way. We emphasize that this class of critical states with $j_{\|}<j_{c \|}$ corresponds to the general situation, while the common Bean critical states and the double critical states are only limiting cases occurring when $j_{\|}=0$ or $j_{\|}=j_{c \|}$, respectively.

Such general critical states, which we shall call the Tcritical states ( $\mathrm{T}$ means transport) $\stackrel{38}{\underline{3}}$ occur even for simple experimental situations. In particular, they appear in a certain region of thin rectangular platelets in a perpendicular magnetic field (in platelets with thickness exceeding the London penetration depth this is the region which is not penetrated by the perpendicular component of the magnetic field) $\stackrel{39}{n}$ Critical states of this type also appear at the vortex-shaking in rectangular platelets $\underline{40}$ and even in strips if the ac field is along the axis of the strips. ${ }^{41}$ They also occur in low-frequency ac experiments with a slab when a circularly polarized ac field is applied perpendicularly to the dc magnetic field $H_{a}$ that is normal to the plane of the slab. $\underline{\underline{42,43}}$
As was pointed out in Refs. 25 27, one more type of critical states can exist in superconductors. In these states $j_{\perp}<j_{c \perp}$ and $j_{\|}=j_{c \|}$, i.e., only flux cutting occurs without any transport of vortices. The description of such C-critical states (C means cutting) in samples of arbitrary shape can be obtained by an immediate generalization of the approach used in Ref. 25,27 for a superconducting slab. Below we shall not analyze such states in detail but only briefly outline this generalization.

In Sec. II of this paper we develop the approach of Ref. 37. In particular, we take into account the dependence of $j_{c \perp}$ on $j_{\|}$and anisotropy of flux-line pinning. We also discuss the relationship between the equations of Ref. 37 and the variational principle recently proposed $\stackrel{44.45 .46}{ }$ In Sec. III we then analyze three examples of the general T-critical state.

\section{GENERAL CRITICAL STATES}

\section{A. Critical-state equations}

The critical state is well established in a sample if the characteristic time of change of the applied magnetic field $\mathbf{H}_{a}, j_{c \perp} d /\left|d \mathbf{H}_{a} / d t\right|$, considerably exceeds the time of flux flow across the sample, $\mu_{0} d^{2} / \rho_{\mathrm{ff}}$, where $d$ is a characteristic size of the sample and $\rho_{\mathrm{ff}}$ is the flux-flow resistivity. In other words, the concept of the critical state can be used for a description of the magnetic-field and current distributions in superconductors if the generated eddy electric fields are relatively small,

$$
\mu_{0} d\left|\frac{d \mathbf{H}_{a}}{d t}\right| \ll \rho_{\mathrm{fff}} j_{c \perp} .
$$

The ideal critical state thus corresponds to the limit $\rho_{\mathrm{ff}} \rightarrow \infty$. Below we imply condition (5) to be fulfilled.

The general T-critical states with $j_{\|}<j_{c \|}$ can be described by the following approach: 37 The static equations (11) and (2) are supplemented by the quasistatic Maxwell equation

$$
\operatorname{rot} \mathbf{E}=-\mu_{0} \dot{\mathbf{H}}
$$

where $\dot{\mathbf{H}} \equiv \partial \mathbf{H} / \partial t$, and $\mathbf{E}$ is the electric field generated by a change of the applied field $\mathbf{H}_{a}$. For the set of equations (11), (2), and (6) to be solvable, it has to be supplemented by the current-voltage law $\mathbf{E}(\mathbf{j}, \mathbf{B}) \stackrel{47}{\underline{47}}$ This law is introduced from two well-known physical ideas: 1 ) At any given $\mathbf{j}$ and $\mathbf{B}$, the direction of $\mathbf{E}$ follows from $\mathbf{E}=[\mathbf{B} \times \mathbf{v}]$, i.e.,

$$
\mathbf{E} \|[\mathbf{B} \times \mathbf{v}],
$$

where $\mathbf{v}$ is the vortex velocity caused by the Lorentz force $[\mathbf{j} \times \mathbf{B}]$. Here for simplicity we shall neglect the so-called Hall angle,,$\frac{48}{,}$ and so the directions of $\mathbf{v}$ and the Lorentz force coincide. 2) The magnitude of $\mathbf{E}$ is found from the condition that

$$
\left|\mathbf{j}_{\perp}\right|=j_{c \perp} .
$$


In fact, this condition may be interpreted as the following current-voltage dependence:

$$
\begin{aligned}
& |\mathbf{E}|=0 \quad \text { at } j_{\perp}<j_{c \perp}, \\
& |\mathbf{E}| \rightarrow \infty \quad \text { at } j_{\perp}>j_{c \perp},
\end{aligned}
$$

which just corresponds to the ideal critical state.

To proceed with our analysis, let us introduce the following notations for the magnetic field $\mathbf{H}(\mathbf{r})$ and the current density $\mathbf{j}(\mathbf{r})$ in the critical state: $\mathbf{H}(\mathbf{r})=H(\mathbf{r}) \boldsymbol{\nu}(\mathbf{r})$, $\mathbf{j}(\mathbf{r})=j(\mathbf{r}) \mathbf{n}(\mathbf{r})$ where $H$ and $j$ are the absolute values of the magnetic field and the current density while the unit vectors $\boldsymbol{\nu}$ and $\mathbf{n}$ define their directions. Then, the component of the current density perpendicular to the magnetic field is given by

$$
\mathbf{j}_{\perp}=\mathbf{j}-\boldsymbol{\nu}(\boldsymbol{\nu} \mathbf{j}) \equiv j_{c \perp} \mathbf{n}_{\perp}(\mathbf{r}) .
$$

Here the unit vector $\mathbf{n}_{\perp}$ defines the direction of $\mathbf{j}_{\perp}$, $\mathbf{n}_{\perp}=(\mathbf{n}-\boldsymbol{\nu}(\boldsymbol{\nu} \mathbf{n})) / D ; D=\sqrt{1-(\mathbf{n} \boldsymbol{\nu})^{2}}$ is the normalizing factor that is equal to the sine of the angle between $\mathbf{H}$ and $\mathbf{j}$, and we have taken into account the condition $\left|\mathbf{j}_{\perp}\right|=j_{c \perp}$. These formulas also lead to the explicit expression for the magnitude $j$ of the current density,

$$
j=\frac{j_{c \perp}(\mathbf{H})}{D},
$$

that is only another form of the condition $\left|\mathbf{j}_{\perp}\right|=j_{c \perp}$. Let us now formulate condition (7). Let at a moment of time $t$ the external magnetic field $\mathbf{H}_{a}(t)$ change infinitesimally by $\delta \mathbf{H}_{a}=\dot{\mathbf{H}}_{a} \delta t$. Under the change of $\mathbf{H}_{a}$, the critical currents locally shift the vortices in the direction of the Lorentz force $[\mathbf{j} \times \boldsymbol{\nu}]$; this shift generates an electric field directed along $[\boldsymbol{\nu} \times[\mathbf{j} \times \boldsymbol{\nu}]]=\mathbf{j}_{\perp}$, i.e., along the vector $\mathbf{n}_{\perp}$. Thus, we can represent the electric field $\mathbf{E}(\mathbf{r})$ in the form:

$$
\mathbf{E}=\mathbf{n}_{\perp} e,
$$

where the scalar function $e(\mathbf{r})$ is the modulus of the electric field. Note that the electric field in general is not parallel to the total current density $\mathbf{j}(\mathbf{r})$. With formulas (10) and (11), equations (11), (2), and (6) are sufficient to describe the T-critical states in a sample of arbitrary shape. It is important that the magnetic fields $\mathbf{H}(\mathbf{r})$ and currents $\mathbf{j}(\mathbf{r})$ in the critical state at the moment of time $t+\delta t$ depends only on the field and current distributions in the previous critical state at the moment $t$ and on the change of the external field $\delta \mathbf{H}_{a}=\dot{\mathbf{H}}_{a} \delta t$, while the electric field $e$ is proportional to the sweep rate $\dot{\mathbf{H}}_{a}$ rather than to $\delta \mathbf{H}_{a}$, and so it plays an auxiliary role in solving the critical-state problem ${ }^{37}$

We emphasize that $e$ is now found as a solution of the set of equations (11), (2), (6), (10), (11) without using the specific current-voltage dependence (9). The explicit equation for the scalar function $e(\mathbf{r})$ has the form: 37

$$
\begin{array}{r}
\mathbf{n}_{\perp} \cdot\{\operatorname{rot} \operatorname{rot}(\mathbf{E})-(\boldsymbol{\nu} \cdot \operatorname{rot} \boldsymbol{\nu}) \operatorname{rot}(\mathbf{E})\}= \\
\frac{\partial j_{c \perp}(\mathbf{H})}{\partial \mathbf{H}} \cdot \operatorname{rot}(\mathbf{E}),
\end{array}
$$

where $\mathbf{E}$ is given by Eq. (11). Continuity of the magnetic field on the surface of the superconductor, $S$, yields the boundary condition to Eq. (12):

$$
-\operatorname{rot}\left(\mathbf{E}\left(\mathbf{r}_{S}\right)\right)=\mu_{0} \dot{\mathbf{H}}_{a}+\int \frac{\left[\mathbf{R} \times \operatorname{rot} \operatorname{rot}\left(\mathbf{E}\left(\mathbf{r}^{\prime}\right)\right)\right]}{4 \pi R^{3}} d \mathbf{r}^{\prime},
$$

where $\mathbf{r}_{S}$ is a point on the surface $S, \mathbf{R} \equiv \mathbf{r}_{S}-\mathbf{r}^{\prime}$, $R=|\mathbf{R}|$, and the integration is carried out over the volume of the sample. The right hand side of this boundary condition expresses $\mu_{0} \dot{\mathbf{H}}$ on the surface of the superconductor (but reaching from outside) with the use of the Biot-Savart law. If in the critical state of the superconductor there are also boundaries at which the direction of the critical currents changes discontinuously or which separate regions with $j_{\perp}=j_{c \perp}$ from regions with $j=0, \underline{49}$ the function $e(\mathbf{r})$ has to vanish at these boundaries to provide continuity of the electric field $e \mathbf{n}_{\perp}$ there.

In practical calculations of critical states developing in the process of changing $\mathbf{H}_{a}(t)$ it is convenient to rewrite Eqs. (10) and (6) in the form

$$
\mu_{0} j[\mathbf{n} \times \dot{\mathbf{n}}]=-[\mathbf{n} \times \operatorname{rot} \operatorname{rot}(\mathbf{E})],
$$

which is a differential equation for the angles defining the direction of $\mathbf{j}$, i.e., the unit vector $\mathbf{n}=\mathbf{j} / j$. Note that since the distributions of the magnetic fields and currents in the critical states of a superconductor are independent of the sweep rate $\dot{\mathbf{H}}_{a}$, their temporal dependence is only a parameterization of their dependence on $\mathbf{H}_{a}$.

Let us now write explicitly the applicability condition of the above theory. Since the projection of $\mathbf{j}$ on the local direction of $\mathbf{H}$ is $j_{c \perp}(\mathbf{n} \boldsymbol{\nu}) / D$, the condition that flux-line cutting is absent leads to the following restriction on the angle between the local $\mathbf{j}$ and $\mathbf{H}$ :

$$
\frac{|\mathbf{n} \boldsymbol{\nu}|}{\sqrt{1-(\mathbf{n} \boldsymbol{\nu})^{2}}}<\frac{j_{c \|}}{j_{c \perp}}
$$

where $j_{c \|}$ is the longitudinal critical current density.

Finally, we make several remarks on the electric field. It may turn out that the electric field $e \mathbf{n}_{\perp}$ obtained with Eq. (12) does not satisfy the condition $\operatorname{div}\left(e \mathbf{n}_{\perp}\right)=0$. To clarify this situation, it is necessary to remember that a moving vortex generates an electric dipolar moment, $\underline{48}$ and hence the moving vortex medium is characterized by the vector of polarization $\mathbf{P}$ which is the macroscopic density of this moment. It follows from the results of Ref. 48 that $\mathbf{P}=-e \mathbf{n}_{\perp}$, and a nonzero $\operatorname{div}\left(e \mathbf{n}_{\perp}\right)$ means that in a type-II superconductor the electric-charge density - $\operatorname{div} \mathbf{P}$ appears which generates a curl-free electric field $\mathbf{E}_{p}=-\nabla \Phi$ described by the scalar potential $\Phi$. This potential field is a part of the total electric field given by $\mathbf{E}=e \mathbf{n}_{\perp}$ inside the sample, and it obeys the equation $\operatorname{div} \mathbf{E}_{p}=\operatorname{div}\left(e \mathbf{n}_{\perp}\right)$, i.e.,

$$
\Delta \Phi=-\operatorname{div}\left(e \mathbf{n}_{\perp}\right),
$$

where $\Delta \equiv \operatorname{div} \nabla$. At the surface of the sample, $S$, the field $\mathbf{E}_{p}$ satisfies the same boundary conditions as in the 
electrostatics of dielectrics: $: \underline{47}$ The tangential components of $\mathbf{E}_{p}$ and the normal component of $\mathbf{E}_{p}+\mathbf{P}=\mathbf{E}_{p}-e \mathbf{n}_{\perp}$ are continuous there. Since $\mathbf{P}=0$ outside the sample, the latter condition means that

$$
\left(\mathbf{E}_{p}^{+}-\mathbf{E}_{p}^{-}\right) \boldsymbol{\tau}=-e \mathbf{n}_{\perp} \boldsymbol{\tau}
$$

where $\mathbf{E}_{p}^{+}$and $\mathbf{E}_{p}^{-}$are the surface potential fields calculated outside and inside $S$, respectively, and $\boldsymbol{\tau}$ is the normal to $S$ pointing outside. The right hand side of Eq. (17) gives the surface-charge density induced by moving vortices in the sample. Note that the potential part of $e \mathbf{n}_{\perp}$ does not influence the magnetic fields and currents in the critical state since $\operatorname{rot} \mathbf{E}_{p}=0$. Appearance of this part is caused by condition (11) that dictates the direction of the electric field in the sample. Although both the inductive part of the electric field, $e \mathbf{n}_{\perp}-\mathbf{E}_{p}$, which generates the critical states, and the potential part $\mathbf{E}_{p}$ can be measured in certain situations, $\stackrel{50}{=}$ we shall not analyze electric fields in detail in this paper since these fields plays only an auxiliary role in the critical state problem. See also the recent book on electric fields 51

Generally speaking, in the process of changing $\mathbf{H}_{a}$ a migration of the induced charges $\rho=\operatorname{div}\left(e \mathbf{n}_{\perp}\right)$ occurs, which leads to a generation of currents satisfying $\operatorname{divj}=-(\partial \rho / \partial t)$ and violating Eq. (2). However, these nonstationary currents are proportional to the second power of the sweep rate $\dot{\mathbf{H}}_{a}$ and are negligible under assumption (5).

\section{B. Generalizations}

We now point out some generalizations of the above results which may be useful in analyzing critical states in real situations.

\section{1. $j_{c \perp}$ depends on $j_{\|}$}

The current-voltage law used in Sec. II A, Eq. (9), means that flux creep is negligible in our approach. In this case the critical current density is found from the condition that the creep activation barrier $U$ of a vortex bundle is equal to zero. It has been implied above that $j_{c \perp}$ may depend on $\mathbf{B}$ but is completely independent of the magnitude of $j_{\|}$. In other words, the form $U=U\left(j_{\perp}, \mathbf{B}\right)$ has been assumed for this $U$. However, the creep activation barrier $U$, generally speaking, may depend not only on $j_{\perp}$ and $\mathbf{B}$ but also on the $j_{\|}$that characterizes flux-line misalignment in the bundle, i.e., in the general case one has $U=U\left(j_{\perp}, j_{\|}, \mathbf{B}\right)$. Then the critical current density $j_{c \perp}$ determined from $U\left(j_{\perp}, j_{\|}, \mathbf{B}\right)=0$ takes the form $j_{c \perp}=j_{c \perp}\left(\mathbf{B}, j_{\|}\right)$. One may expect that this dependence of $j_{c \perp}$ on the longitudinal current component $j_{\|}$is especially noticeable when $j_{\|}$ is close to its critical value $j_{c \|}$, and hence $j_{c \perp}\left(\mathbf{B}, j_{c \|}\right)$ in general differs from $j_{c \perp}(\mathbf{B}, 0)$. Similarly, the activation barrier $U_{\text {cut }}$ for flux cutting is a function of both currentdensity components and of the magnetic induction, i.e., $U_{\text {cut }}=U_{\text {cut }}\left(j_{\perp}, j_{\|}, \mathbf{B}\right)$, and the condition $U_{\text {cut }}=0$ gives $j_{c \|}=j_{c \|}\left(\mathbf{B}, j_{\perp}\right)$. In Fig. 1a at a fixed $\mathbf{B}$ we schematically show the dependences of $j_{c \perp}$ on $j_{\|}$and of $j_{c \|}$ on $j_{\perp}$ in the plane $\left(j_{\perp}, j_{\|}\right)$. Note that these dependences cross when the equations $U\left(j_{\perp}, j_{\|}, \mathbf{B}\right)=0$ and $U_{\text {cut }}\left(j_{\perp}, j_{\|}, \mathbf{B}\right)=0$ hold simultaneously. This occurs at isolated points in the $j_{\perp-} j_{\|}$plane since the barriers $U$ and $U_{\text {cut }}$ characterize different physical processes and are essentially different functions of the current components. These points correspond to the double critical states when $j_{\perp}=j_{c \perp}$ and $j_{\|}=j_{c \|}$. In Fig. 1a the top/bottom and right/left sections of the curves between the four points describe $j_{c \perp}\left(j_{\|}\right)$in the general T-critical state and $j_{c \|}\left(j_{\perp}\right)$ in the C-critical state.

The dependence $j_{c \perp}\left(j_{\|}\right)$leads to a replacement of $j_{c \perp}(\mathbf{H})$ by $j_{c \perp}\left(\mathbf{H}, j_{\|}\right)$in formula (10) that now reads

$$
j D=j_{c \perp}\left(\mathbf{H}, j \sqrt{1-D^{2}}\right) .
$$

The dependence $j_{c \perp}\left(j_{\|}\right)$also leads to a modification of Eq. (12). In the right hand side of this equation the term $-\mu_{0}\left(\partial j_{c \perp} / \partial j_{\|}\right)\left(\partial j_{c \|} / \partial t\right)$ should be added that equals

$$
\frac{\partial j_{c \perp}}{\partial j_{\|}}\left(\frac{j_{c \perp}}{H} \mathbf{n}_{\perp} \cdot \operatorname{rot}(\mathbf{E})+\boldsymbol{\nu} \cdot \operatorname{rot} \operatorname{rot}(\mathbf{E})\right)
$$

with $\mathbf{E}$ from Eq. (11). Note that the first term in this expression has no singularity at $H \rightarrow 0$ since the combination $\mathbf{n}_{\perp} \cdot \operatorname{rot}\left(e \mathbf{n}_{\perp}\right)$ can be also rewritten as $e \mathbf{n}_{\perp} \cdot \operatorname{rot} \mathbf{n}_{\perp}$ and $e=|[\mathbf{B} \times \mathbf{v}]| \propto H$.

In Refs. 52,53 a phenomenological model of the general critical state was considered that described sufficiently well a number of experimental data on the magnetization of a slab and of a disk in magnetic fields parallel to their planes. In fact, in this model a certain type of the dependence of $j_{c \perp}$ on $j_{\|}$(and of $j_{c \|}$ on $j_{\perp}$ ) was introduced. Even though the directions of the electric field in this model do not satisfy the physical requirement (11), the sufficiently good description of the data seems to indicate the importance of this dependence in real situations.

\section{Anisotropy of flux-line pinning}

In deriving Eq. (11) we have assumed that when $\mathbf{H}_{a}$ changes, vortices shift in the direction of the local Lorentz force $[\mathbf{j} \times \mathbf{B}]$. However, in the case of anisotropic pinning this assumption may fail. Nevertheless, even in this case the direction of the shift can be expressed via the directions of $\mathbf{j}$ and $\boldsymbol{\nu} \equiv \mathbf{H} / H$, see Appendix A in Ref. 39. Now the unit vector $\mathbf{u}$ along the electric field, $\mathbf{E}=\mathbf{u} e$, is

$$
\mathbf{u}=\mathbf{n}_{\perp} \cos \delta+\left[\boldsymbol{\nu} \times \mathbf{n}_{\perp}\right] \sin \delta,
$$

where the angle $\delta$ describes the change of the direction of the electric field due to anisotropic pinning. If in the plain perpendicular to the local $\mathbf{H}$ the critical current 

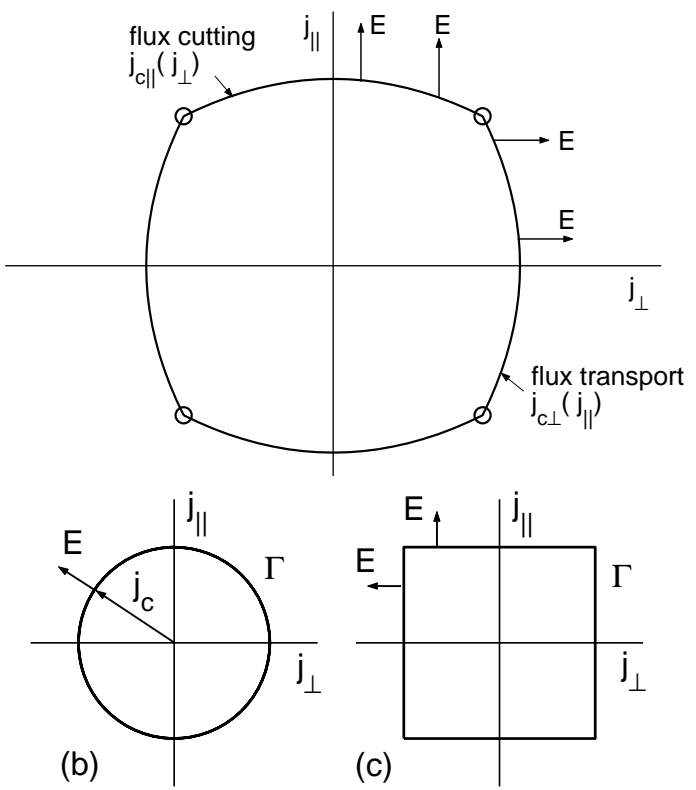

FIG. 1: (a): Dependences of $j_{c \perp}$ on $j_{\|}$and of $j_{c \|}$ on $j_{\perp}$ (solid lines) shown schematically in the $j_{\perp}-j_{\|}$plane at a fixed $\mathbf{B}$, see Sec. II B1. The crossing points of the lines correspond to the double critical states when $j_{\perp}=j_{c \perp}$ and $j_{\|}=j_{c \|}$. Shown are also the directions of the electric field for the appropriate critical states. (b) and (c): The boundaries $\Gamma$ (solid lines) of the regions $\Delta$ introduced by Badía and López ${ }^{44,45,46}$ in the $j_{\perp}-j_{\|}$plane, see Sec. II C. Here the regions $\Delta$ are (b) a circle and (c) a rectangle. Shown are also the directions of the electric field according to the approach of Badía and López.

density $j_{c \perp}$ depends on its direction $\mathbf{n}_{\perp}$, the angle $\delta$ is found from ${ }^{39}$

$$
\tan \delta=-\frac{\partial\left[\ln j_{c \perp}(\mathbf{B}, \phi)\right]}{\partial \phi},
$$

where $\phi$ is the angle defining the direction of $\mathbf{n}_{\perp}=$ $(\cos \phi, \sin \phi)$ in the plane perpendicular to $\mathbf{H}$. When $j_{c \perp}$ is isotropic in this plane, we obtain $\delta=0$, and thus $\mathbf{u}$ coincides with $\mathbf{n}_{\perp}$.

Equations (20) and (21) give a relation between $\mathbf{n}_{\perp}$ and $\mathbf{u}$. When $\delta \neq 0$, i.e., when the vector $\mathbf{u}$ differs from $\mathbf{n}_{\perp}$, the only change in the critical state equations is that $e \mathbf{n}_{\perp}$ in Eq. (11) is replaced by $e \mathbf{u}$, and $j_{c \perp}(\mathbf{H})$ in Eq. (10) is now $j_{c \perp}\left(\mathbf{H}, \mathbf{n}_{\perp}\right)$.

\section{3. $C$-critical states}

As it was mentioned in the Introduction, in the case of an infinite slab the critical states with flux-line cutting but without flux-line transport were considered in Refs. 25,27. For samples of arbitrary shape such Ccritical states can be described by Eqs. (11), (2), and (6), but now the electric field is along the local $\mathbf{H}$, i.e., $\mathbf{E}=\boldsymbol{\nu} e$. This condition replaces Eq. (7) [or (11)]. The absolute value $e$ of the electric field is now determined by the condition $j_{\|}=j_{c \|}$ which is equivalent to the following current-voltage dependence:

$$
\begin{aligned}
& |\mathbf{E}|=0 \quad \text { at } j_{\|}<j_{c \|}, \\
& |\mathbf{E}| \rightarrow \infty \quad \text { at } j_{\|}>j_{c \|},
\end{aligned}
$$

and leads to the formula

$$
j=\frac{j_{c \|}(\mathbf{H})}{|\mathbf{n} \nu|} .
$$

Equations (22), (23) replace Eqs. (91) and (10), respectively.

\section{Variational principle}

Recently, $\stackrel{44,45,46}{\longleftarrow}$ a variational principle was put forward to describe the critical states in superconductors. In deriving this principle Badía and López used Eqs. (10), (6) and the current-voltage law with $|\mathbf{E}|=0$ when $\mathbf{j}$ is inside some region $\Delta$ of the $\mathbf{j}$-space and $|\mathbf{E}| \rightarrow \infty$ when $\mathbf{j}$ lies outside this region. In other words, the critical states correspond to the boundary $\Gamma$ of the region $\Delta$, see Fig. 1b,c. However, the physical idea of the direction of the electric field, Eq. (7), was not incorporated in their principle. Instead of this they find the direction of the electric field from some condition of maximality of their Hamiltonian. This leads them to the conclusion that the electric fields in the critical states are directed along the normals to the boundary $\Gamma$ at the appropriate points, Fig. 1b,c.

Within our approach their boundary $\Gamma$ corresponds to the contour composed of the dependences $j_{c \perp}\left(j_{\|}\right)$and $j_{c \|}\left(j_{\perp}\right)$, see Fig. 1a and Sec. II B1. But in our general T-critical states with $j_{\|}<j_{c \|}$ the electric field is always perpendicular to the local $\mathbf{H}$ (i.e., to $\mathbf{j}_{\|}$), and in the Ccritical states with flux-line cutting but without flux-line transport the electric field is along the local $\mathbf{H}$. It is clear that only in the case when $\Delta$ is a rectangle does the approach of Badía and López lead to the correct results for the electric field, Fig. 1 $\stackrel{54}{t}$ However, in general their approach leads to contradiction with existing physical concepts $2,23,24$ In particular, in the so-called isotropic model, when $\Delta$ is a circle, Fig. $1 b$, the electric field $\mathbf{E}$ is parallel to $\mathbf{j}$, and hence a nonzero $\mathbf{E}$ along $\mathbf{H}$ appears even for an infinitesimally small longitudinal component of $\mathbf{j}$, i.e., flux-line cutting in that model occurs without any threshold $j_{c \|}, \underline{55}$

\section{EXAMPLES}

We first consider two examples of the general critical state in an infinite slab of thickness $d$. Let this slab fill the space $|x|,|y|<\infty,|z| \leq d / 2$, and be in a constant and uniform external magnetic field $H_{a z}$ directed along the $z$ axis, i.e., perpendicularly to the slab plane. The critical current density $j_{c \perp}$ is assumed to be constant in this slab. In the first example a constant field $H_{a x}$ 
$\left(H_{a x} \geq J_{c} / 2=d j_{c \perp} / 2\right)$ is applied along the $x$ axis, and after that the magnetic field $H_{a y}$ is switched on in the $y$ direction. This example was considered in our paper,, 37 but there $H_{a x}, H_{a y}$, and $J_{c}$ were assumed to be small as compared with $H_{a z}$, i.e., the tilt angle $\theta$ of the magnetic field to the $z$ axis was always small. Now we do not put this restriction, and the angle $\theta$ may be sufficiently large. But we still assume that flux-line cutting does not occur (see below). This example may be considered as a modification of the experimental conditions of Refs. 34 where the suppression of the magnetic moment of the slab was investigated at $H_{a z}=0$. In the second example the critical current along the $y$ axis is applied to a slab, and after that the magnetic field $H_{a y}$ is switched on in the same direction.

The critical state equations are the same for these two examples. The difference is only in the boundary conditions. Let us write these equations. The condition $\operatorname{div} \mathbf{j}=\partial j_{z} / \partial z=0$ together with $\left.j_{z}\right|_{z= \pm d / 2}=0$ yields $j_{z}=0$, i.e., the currents flow in the $x-y$ planes $\underline{56}$ Then, to describe the critical state, we may use the parameterization:

$$
\begin{array}{r}
\mathbf{j}=j_{c}(\varphi, \theta, \psi)(\cos \varphi(z), \sin \varphi(z), 0), \\
\mathbf{H}(z)=\hat{\mathbf{z}} H_{a z}+\mathbf{h}(z), \\
\mathbf{h}(z)=\left(h_{x}(z), h_{y}(z), 0\right),
\end{array}
$$

where $\hat{\mathbf{z}}$ is the unit vector along the $z$ axis; $j_{c}(\varphi, \theta, \psi)$ is the magnitude of the critical current density when a flux-line element is given by the angles $\psi$ and $\theta$, $\tan \psi=h_{y} / h_{x}, \tan \theta=\left(h_{x}^{2}+h_{y}^{2}\right)^{1 / 2} / H_{a z}$, while the current flows in the direction defined by the angle $\varphi$; all these angles generally depend on $z$. A dependence of $j_{c}$ on the orientation of the local $\mathbf{H}$ appears even at a constant $j_{c \perp}$ if $j_{c}$ is not perpendicular to this $\mathbf{H}$, and this dependence is described by formula (10), where $D$ in terms of the angles is

$$
D=\left[1-\cos ^{2}(\varphi-\psi) \sin ^{2} \theta\right]^{1 / 2} .
$$

With this parameterization, the equation $\operatorname{div} \mathbf{H}=0$ is satisfied identically, while the Maxwell equation $\operatorname{rot} \mathbf{H}=\mathbf{j}$ reads

$$
\begin{aligned}
\frac{d h_{x}}{d z} & =\frac{j_{c \perp} \sin \varphi}{D}, \\
-\frac{d h_{y}}{d z} & =\frac{j_{c \perp} \cos \varphi}{D} .
\end{aligned}
$$

These equations differ from the appropriate equations of Ref. 37 by the factor $1 / D$, which is not unity now.

In the case under study one has $\mathbf{n}=(\cos \varphi, \sin \varphi, 0)$, $\boldsymbol{\nu}=(\sin \theta \cos \psi, \sin \theta \sin \psi, \cos \theta)$. Then, a direct calculation gives the following expressions for the vector $\mathbf{n}_{\perp}$ defining the direction of the current component perpendicular to $\mathbf{H}$ :

$$
n_{\perp x}=\frac{\cos \varphi-\sin ^{2} \theta \cos \psi \cos (\varphi-\psi)}{D},
$$

$$
\begin{aligned}
& n_{\perp y}=\frac{\sin \varphi-\sin ^{2} \theta \sin \psi \cos (\varphi-\psi)}{D}, \\
& n_{\perp z}=-\frac{\sin \theta \cos \theta \cos (\varphi-\psi)}{D},
\end{aligned}
$$

and equation (12) for the electric field $e$ takes the form:

$$
\begin{aligned}
n_{\perp x}\left(e n_{\perp x}\right)^{\prime \prime} & +n_{\perp y}\left(e n_{\perp y}\right)^{\prime \prime} \\
& -\psi^{\prime} \sin ^{2} \theta\left(n_{\perp x}^{\prime} n_{\perp y}-n_{\perp x} n_{\perp y}^{\prime}\right) e=0,
\end{aligned}
$$

where the dash over a symbol means $\partial / \partial z$. For the angle $\varphi$ we obtain from Eq. (14):

$$
\mu_{0} \frac{j_{c \perp}}{D} \frac{\partial \varphi}{\partial t}=\left(e n_{\perp y}\right)^{\prime \prime} \cos \varphi-\left(e n_{\perp x}\right)^{\prime \prime} \sin \varphi .
$$

At small $\theta$ when $D \approx 1$ and $\mathbf{n}_{\perp} \approx \mathbf{n}$, equations (29) and (30) reduce to the form that was used in Ref. 37:

$$
\begin{aligned}
e^{\prime \prime}-\left(\varphi^{\prime}\right)^{2} e & =0, \\
\mu_{0} j_{c \perp} \frac{\partial \varphi}{\partial t} & =2 e^{\prime} \varphi^{\prime}+e \varphi^{\prime \prime} .
\end{aligned}
$$

Equations (26)-(30) provide the complete description of any general T-critical state in an infinite slab with $j_{c \perp}(\mathbf{H})=$ constant in the absence of flux-line cutting. For different critical-state problems only the boundary conditions should be appropriately chosen. Note that the usual Bean critical states in the slab correspond to discontinuous solutions $\varphi(z)$ of these equations.

In the case of the slab condition (15) of absence of flux-line cutting leads to the following restriction on the angles $\theta, \psi$, and $\varphi$ :

$$
\frac{\sin \theta|\cos (\varphi-\psi)|}{D}<\frac{j_{c \|}}{j_{c \perp}} .
$$

This condition is fulfilled at any $\varphi$ and $\psi$, i.e., at any direction of $\mathbf{j}$ and $\mathbf{h}$, if the $z$ component of the magnetic field, $H_{a z}$, is not too small,

$$
\tan \theta=\frac{\sqrt{h_{x}^{2}+h_{y}^{2}}}{H_{a z}}<\frac{j_{c \|}}{j_{c \perp}} .
$$

We imply this condition to be fulfilled below.

\section{A. First example: $H_{a x}$ and $H_{a y}$}

In the first example that we consider, a constant field $H_{a x}\left(H_{a x} \geq J_{c} / 2=d j_{c \perp} / 2\right)$ is applied along the $x$ axis, and after that the magnetic field $H_{a y}$ is switched on in the $y$ direction. Then, the boundary conditions to Eqs. (26) - (30) at $z=d / 2$ are

$$
\begin{array}{r}
h_{x}=H_{a x}, \quad h_{y}=H_{a y}(t), \\
\left(e n_{\perp x}\right)^{\prime}=-\mu_{0} \frac{d H_{a y}(t)}{d t}, \quad\left(e n_{\perp y}\right)^{\prime}=0,
\end{array}
$$


or equivalently, conditions (36), which follow from formula (13), can be rewritten in the form:

$$
\begin{gathered}
e^{\prime}\left(n_{\perp x}^{\prime} n_{\perp y}-n_{\perp x} n_{\perp y}^{\prime}\right)=\mu_{0} \frac{d H_{a y}(t)}{d t} n_{\perp y}^{\prime}, \\
e\left(n_{\perp x}^{\prime} n_{\perp y}-n_{\perp x} n_{\perp y}^{\prime}\right)=-\mu_{0} \frac{d H_{a y}(t)}{d t} n_{\perp y} .
\end{gathered}
$$

Taking into account the symmetry of the problem, $e(-z)=e(z), \varphi(-z)=\varphi(z)-\pi, \mathbf{h}(-z)=\mathbf{h}(z)$, it is sufficient to solve equations (26)-(30) in the region $0 \leq z \leq d / 2$. At $z=0$, where the direction of the currents changes discontinuously, one has the additional condition for $e$,

$$
e(0)=0 \text {. }
$$

Since after switching on $H_{a x}$, the critical currents flow in the $y$ direction, we have the following initial condition for Eq. (30):

$$
\varphi(z, t=0)=\pi / 2,
$$

where the moment $t=0$ corresponds to the beginning of switching on $H_{a y}$. As to the initial magnetic-field profiles, equations (26), (27), and (39) give $h_{y}(z, t=0)=$ $H_{a y}=0$ and $h_{x}(z, t=0)=H_{a x}-0.5 J_{c}+j_{c \perp} z$ where $J_{c} \equiv j_{c \perp} d$.

In the limiting case $H_{a z} \gg H_{a x}, H_{a y}, J_{c}$, the solution of equations (26)-(30) with conditions (35) - (39) was investigated in Ref. 37. Since in this case $\mathbf{n}_{\perp} \approx \mathbf{n}$, one finds that the electric field $e \mathbf{n}_{\perp}$ is along the current density $j \mathbf{n}$, and in fact, we arrive at a situation which can be formally described by the so-called isotropic model of Badía and López. $\stackrel{45}{*}$ As was explained in Sec. II C, this model in general does not lead to the correct direction of the electric field. In particular, it fails in the following situation discussed by Badía and López: $\frac{45}{}$ A slab with $H_{a z}=0, H_{a x}=$ constant, and oscillating $H_{a y}$. But in the case $H_{a z} \gg H_{a x}, H_{a y}, J_{c}$, which in reality was not considered in Ref. 45, the isotropic model gives the correct results, and the numerical data of Ref. 45 agree $\frac{57}{3}$ with those of Ref. 37 and can be used to describe this limiting situation.

In the case $H_{a z} \sim H_{a x}, H_{a y}, J_{c}$, the solution of equations (26)-(30) with conditions (35) - (39) is shown in Fig. 2. We present $\varphi(z), \psi(z), h_{x}(z), h_{y}(z)$ in the sequence of the critical states developed in the process of increasing $H_{a y}$. We do not show the electric field $e$ that is proportional to the sweep rate $\dot{H}_{a y}$ and plays an auxiliary role. As was noticed previously,, 37 in stark contrast to the Bean critical states, in which any change of the current direction occurs inside a narrow front, in the general Tcritical state the change of the angle $\varphi(z)$ with increasing $H_{a y}$ has diffusive character. But there is a difference between the data of Fig. 2 and the results $\frac{37}{3}$ obtained in the case $H_{a z} \gg H_{a x}, H_{a y}, J_{c}$ when the currents in the critical state are almost perpendicular to the local magnetic fields. In the latter case at $H_{a y}>J_{c}$ the field profile

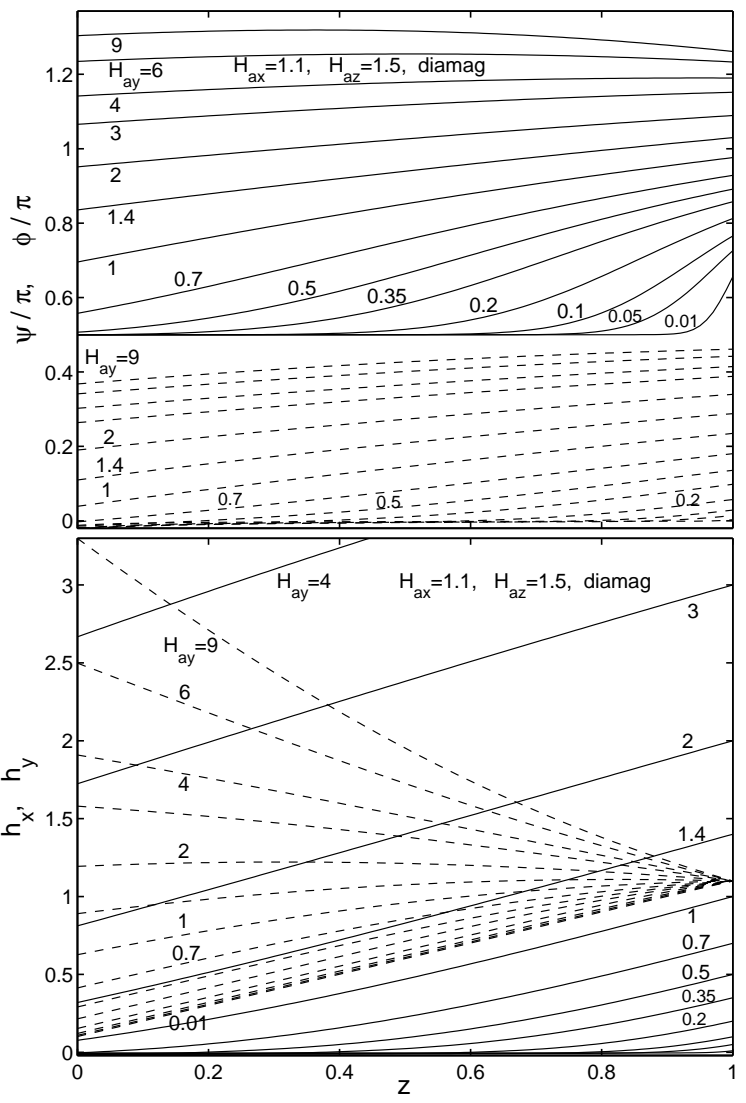

FIG. 2: Top: Profiles of the angle of the currents $\varphi(z)$ (solid lines), and of the field angle $\psi(z), \tan \psi=h_{y}(z) / h_{x}(z)$ (dashed lines) in the critical states of the slab at $H_{a z}=1.5$, $H_{a x}=1.1$ and $H_{a y}=0.01,0.05,0.1,0.2,0.35,0.5,0.7,1$, 1.4, 2, 3, 4, 6, 9. Bottom: The magnetic field components $h_{x}(z)$ (dashed lines) and $h_{y}(z)$ (solid lines) in the same critical states. We start from the diamagnetic initial critical state with $h_{x}(z)=H_{a x}-1+z, h_{y}(z)=H_{a y}=0$, and $\varphi(z)=\pi / 2$. Here $z$ is in units of $d / 2$, and the magnetic fields in units of $j_{c \perp} d / 2=J_{c} / 2$.

$h_{x}(z)$ becomes practically constant and coincides with $H_{a x}$, while the angle $\varphi$ tends to $\pi$. On the other hand, we see from Fig. 2 that for $H_{a z} \sim J_{c}$ the angle $\varphi$ lies in the interval $\pi<\varphi<3 \pi / 2$ at $H_{a y}>J_{c}$. In other words, the $y$ component of $\mathbf{j}(z)$ has the opposite direction as compared with the initial state. This leads to the fact that at $H_{a y}>J_{c}$ the field $h_{x}$ increases towards the central plane of the slab, $z=0$ (but $h(z)=\sqrt{h_{x}^{2}+h_{y}^{2}}$ still decreases towards this plane), and the initial diamagnetic state with the magnetic moment $M_{x}=-j_{c \perp} d^{2} / 4$ (per unit area) turns into a paramagnetic state with positive $M_{x}$.

In Fig. 3 we show the same sequence of the critical states but in the case of the paramagnetic initial state. This initial state is obtained if one first increases the field $H_{a x}$ essentially above the field of full flux penetration and then decreases it to a prescribed value. Now the initial 


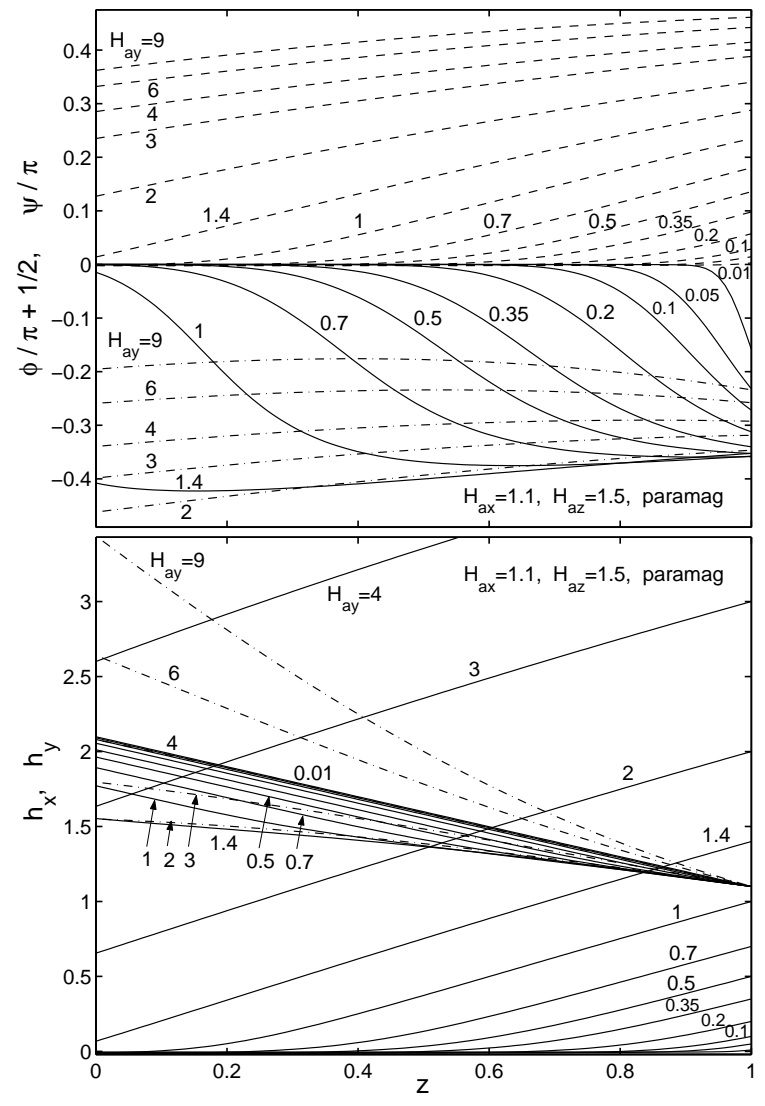

FIG. 3: The same as Fig. 2, but at $H_{a y}=0$ we start from the paramagnetic initial critical state with $h_{x}(z)=H_{a x}+1-z$, $h_{y}(z)=0$, and $\varphi(z)=-\pi / 2$. For clarity the profiles $\varphi(z)$ and $h_{x}(z)$ at $H_{a y} \geq 2$ are shown by dash-dotted lines (and for $H_{a y} \leq 1.4$ by solid lines).

condition to Eq. (30) is

$$
\varphi(z, t=0)=-\pi / 2
$$

and the magnetic fields at $t=0$ are given by $h_{y}(z, t=$ $0)=H_{a y}=0, h_{x}(z, t=0)=H_{a x}+0.5 J_{c}-j_{c \perp} z$. It is seen from Fig. 3 that although at $H_{a y}<H_{a z}$ a decay of the initial paramagnetic profile $h_{x}(z)$ occurs, with a further increase of $H_{a y}$ new paramagnetic states are developed that are close to the appropriate states of Fig. 2.

In Fig. 4 we compare the $H_{a y}$-dependences of the magnetic moment $\left(M_{x}, M_{y}\right)$ per unit area of the slab, $\frac{58}{5}$

$$
\mathbf{M}=\int_{-d / 2}^{d / 2} z[\hat{\mathbf{z}} \times \mathbf{j}] d z,
$$

obtained using the two sequences of the profiles $\mathbf{j}\left(z, H_{a y}\right)$ developed from the diamagnetic and paramagnetic initial states with the same $H_{a x}$. It is seen that in both cases $\left|M_{x}\right|$ and $\left|M_{y}\right|$, and even more $M=\sqrt{M_{x}^{2}+M_{y}^{2}}$, can exceed the "saturation value" $j_{c \perp} d^{2} / 4$ used as unit in Fig. 4. This is possible since the current density $j$ exceeds $j_{c \perp}$ when it does not flow at a right angle to the vortices.
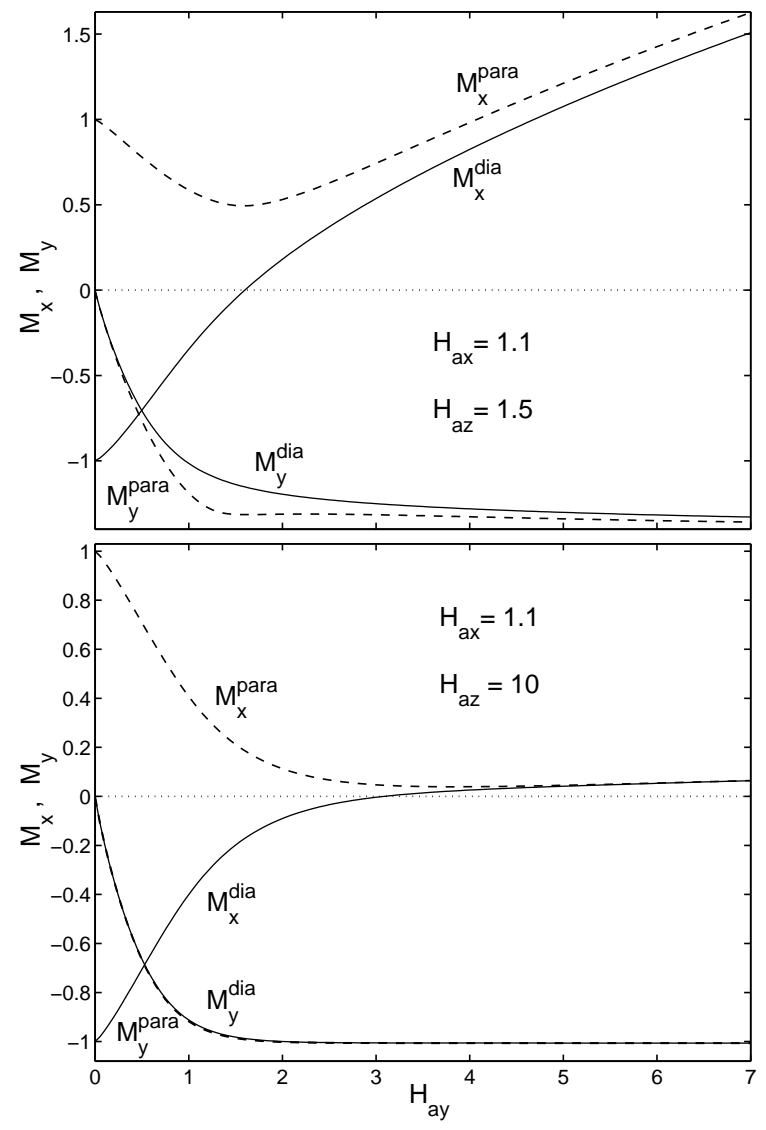

FIG. 4: The magnetic moment $\left(M_{x}, M_{y}\right)$ of the slab per unit area defined by Eq. (41) as a function of $H_{a y}$. Shown are the diamagnetic (solid lines, see Fig. 2) and paramagnetic (dashed lines, see Fig. 3) cases for $H_{a x}=1.1$ and $H_{a z}=1.5$ (top) and $H_{a z}=10$ (bottom). Units are $j_{c \perp} d / 2$ for $H$ and $j_{c \perp} d^{2} / 4$ for $M$.

This excess of $j$ leads to that $M_{x}\left(H_{a y}\right)$ does not saturate at large $H_{a y}$ but continues to increase nearly linearly, with slightly negative curvature. The other component, $M_{y}\left(H_{a y}\right)$, at large $H_{a y}$ practically saturates to a value slightly lower than $-j_{c \perp} d^{2} / 4$. Of course, one should keep in mind that in reality the region of large $H_{a y}$ where these results for $\mathbf{M}$ are applicable is limited by condition (34). Note also that in agreement with Figs. 2 and 3 the magnetic moment $M_{x}\left(H_{a y}\right)$ is always positive at sufficiently large $H_{a y}$, and the diamagnetic and paramagnetic initial states lead to practically the same $M_{x}$ at such $H_{a y}$.

As it is known, field-cooled type-II superconducting samples frequently exhibit a positive magnetic moment; see, e.g., paper ${ }^{59}$ and references therein. Different explanations of this paramagnetic effect were put forward. In particular, this effect may be associated with the compression of trapped magnetic flux in the sample .60 The data of Figs. 2-4 show that in principle, the paramagnetic effect may be also due to the field-cooling caused generation of critical states in which the circulating currents are not perpendicular to the local magnetic fields.

The general T-critical states considered here can be re- 

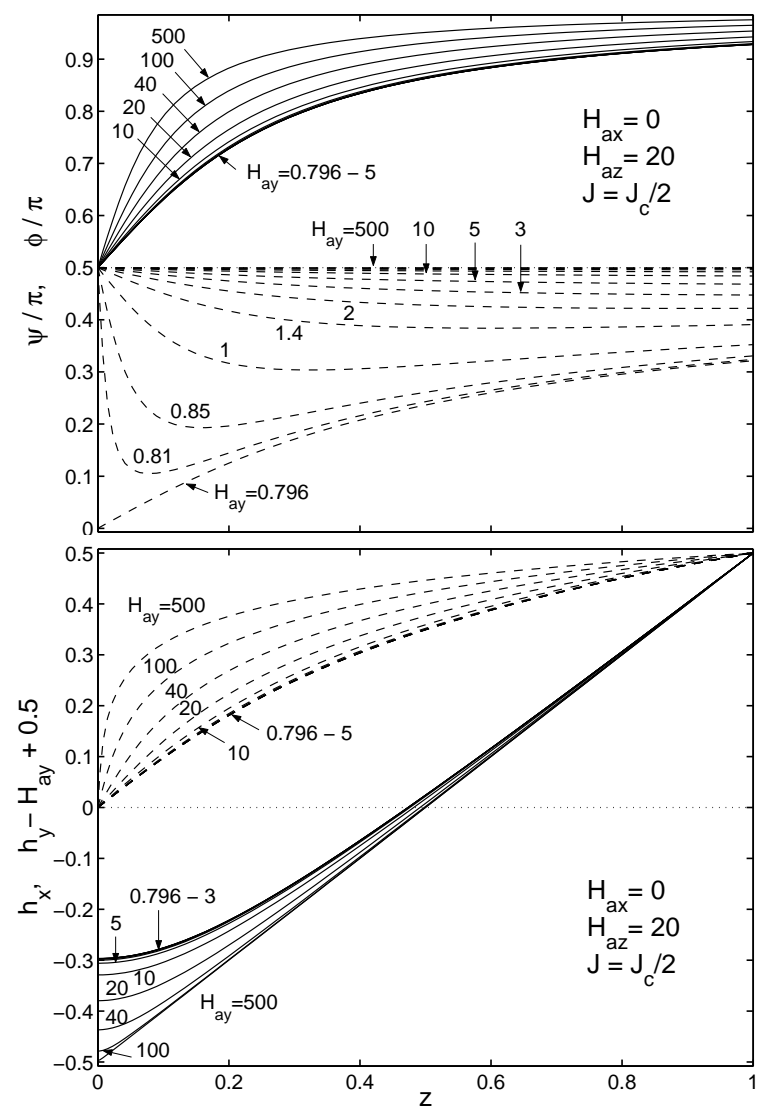

FIG. 5: Profiles of the angles $\varphi(z)$ (solid lines) and $\psi(z)$ (dashed lines) (top), and of the magnetic field components $h_{x}(z)$ (dashed lines) and $h_{y}(z)$ (solid lines) (bottom) for a slab with applied sheet current $J=J_{c} / 2$ and $H_{a x}=0$. The profiles are calculated from Eqs. (45) and (46) for $H_{a y}=0.796$, $0.81,0.85,1,1.4,2,3,5,10,20,40,100,500$ and large $H_{a z}=20$ (all the magnetic fields are in unit of $J_{c} / 2$ ). $H_{a y}^{0}=0.796$ is the field of full flux penetration at $J=J_{c} / 2$.

alized in experiments similar to the experiments of Park et al. $\underline{\underline{ }}$ and Fisher et al. $\stackrel{34}{,}$ except that now the field $H_{a z}$ perpendicular to the plane of the sample is not equal to zero. Such investigations would enable one to compare the theoretical results for the general T-critical states with the appropriate experimental data avoiding complications due to flux-line cutting. To prepare the initial state which was described above, e.g., in a superconducting strip of length $2 L$ and width $2 w$ considerably exceeding its thickness $d, 2 L>2 w \gg d$, one may apply first the field $H_{a z}$ perpendicular to the plane of the strip, and then an oscillating in-plane magnetic field $H_{a x}$ across the width of the strip. This "shaking" leads to a homogeneous distribution of the perpendicular field $H_{a z}$ over the sample ${ }^{62}$ After this shaking process one keeps $H_{a x}=$ constant and applies the field $H_{a y}$ along the axis of the strip.

\section{B. Second example: $J_{y}$ and $H_{a y}$}

We now consider the second example of the general Tcritical state in the slab. It is assumed that the slab is in a uniform magnetic field $H_{a z}$ along the $z$ axis, the current $J$ (per unit length along $x$ ) flows in the $y$ direction, and at $t=0$ the field $H_{a y}$ is switched on. In this case the boundary conditions at $z=d / 2$ are

$$
\begin{array}{r}
h_{x}=\frac{J}{2}, \quad h_{y}=H_{a y}(t), \\
\left(e n_{\perp x}\right)^{\prime}=-\mu_{0} \frac{d H_{a y}(t)}{d t}, \quad\left(e n_{\perp y}\right)^{\prime}=0 .
\end{array}
$$

The symmetry of the problem is now described by the relationships: $e(-z)=e(z), \varphi(-z)=\pi-\varphi(z), h_{x}(-z)=$ $-h_{x}(z), h_{y}(-z)=h_{y}(z)$, and at $z=0$ the direction of the critical currents changes continuously. Thus, instead of condition (38) we have at $z=0$,

$$
h_{x}(0)=0, \quad \varphi(0)=\pi / 2 .
$$

As in the first example, we shall consider the critical states only in the interval $0 \leq z \leq d / 2$.

If $J$ is less than $J_{c}=j_{c \perp} d$, in the initial state the current flows only at $1-\left(J / J_{c}\right) \leq 2 z / d \leq 1$. After switching on $H_{a y}$ the current distribution develops over the whole thickness $d$ when $H_{a y}$ reaches a penetration field $H_{a y}^{0}<J_{c} / 2$, and we shall analyze the critical states only after this penetration of the current has occurred, i.e., at $H_{a y} \geq H_{a y}^{0}$.

Below we consider only the case $H_{a z} \gg J_{c}$. In this case in the leading order in the small parameter $J_{c} / H_{a z}$ we find the following analytic solution of Eqs. (26) - (30) with boundary conditions (42)-(44), see also Fig. 5:

$$
\begin{aligned}
e & =\frac{\mu_{0} \dot{H}_{a y} a D}{\sin \varphi \cos \theta}, \quad \cot \varphi=-\frac{z \cos \theta}{a}, \\
h_{x} & =\frac{j_{c \perp} a}{\cos \theta} \ln \left(\frac{z}{a}+\sqrt{1+\frac{z^{2}}{a^{2}}}\right), \\
h_{y} & =H_{a y}-j_{c \perp} a\left(\sqrt{1+\frac{d^{2}}{4 a^{2}}}-\sqrt{1+\frac{z^{2}}{a^{2}}}\right),
\end{aligned}
$$

where $D^{2}=1-\cos ^{2}(\varphi-\psi) \sin ^{2} \theta \approx 1-\sin ^{2} \varphi \sin ^{2} \theta$ (since either $\psi \approx \pi / 2$ or $\left.\sin ^{2} \theta \ll 1\right), \cos ^{2} \theta \approx H_{a z}^{2} /\left(H_{a z}^{2}+H_{a y}^{2}\right)$, and the length $a$ is determined by the sheet current $J$ and $\cos \theta$,

$$
\frac{J}{J_{c}} \cos \theta=\frac{2 a}{d} \ln \left(\frac{d}{2 a}+\sqrt{1+\frac{d^{2}}{4 a^{2}}}\right)=\frac{2 a}{d} \operatorname{arcsinh} \frac{d}{2 a} .
$$

We shall denote the solution of Eq. (46) as $2 a / d=$ $g\left(J \cos \theta / J_{C}\right)$. The function $g(u)$ defined by $u=$ $g \operatorname{arcsinh}(1 / g)$ increases monotonically with its argument $u$, Fig. 6 . Hence with increasing $H_{a y}$, i.e., with decreasing $\cos \theta$, the length $a$ decreases. When $J$ is close to $J_{c}$ and $\cos \theta \approx 1$, the length $a$ tends to $\infty$, while for $J \ll J_{c}$ one 
has $2 a / d \sim J \cos \theta / J_{c} \ll 1$. A very good approximation valid at all $u$ is $\underline{\underline{41}}$

$$
\begin{aligned}
g(u) & \approx u / \ln \frac{\left(1+g_{0}^{2}\right)^{1 / 2}+1}{g_{0}}, \\
g_{0}(u) & =\frac{u+u^{2}}{[24(1-u)]^{1 / 2}} .
\end{aligned}
$$

The field of full flux penetration can be estimated from $h_{y}(0)=0$,

$$
H_{a y}^{0}=j_{c \perp} a\left(\sqrt{1+\frac{d^{2}}{4 a^{2}}}-1\right),
$$

where $a$ is determined by Eq. (46). When $H_{a y} \ll H_{a z}$, one has $\cos \theta \approx 1$, and the length $a$ is almost independent of $H_{a y}$. Thus, at such $H_{a y}$ the profiles $\varphi(z), h_{x}(z)$, and $h_{y}(z)-h_{a y}$ practically do not change with increasing $H_{a y}$. Fig. 5 shows that this property of the profiles, in fact, holds in the region $H_{a y}<H_{a z} / 2$ when $J / J_{c}=0.5$. However, if $J$ is close to $J_{c}$, the length $a$ sharply depends on $\cos \theta$, Fig. 6 , and the width of this region shrinks.

Solution (45) can be obtained as follows: We put

$$
\left(e n_{\perp x}\right)^{\prime \prime}=0, \quad\left(e n_{\perp y}\right)^{\prime \prime}=0,
$$

since it may be verified that the term proportional to $\psi^{\prime} \sin ^{2} \theta$ in Eq. (29) and the left hand side of Eq. (30) are small in the parameter $J_{c} / H_{a z}$ and hence may be omitted in the first approximation. Equations (49) mean that $e n_{\perp x}$ and $e n_{\perp y}$ are linear functions of $z$, and thus each of them generally depends on two constants. However, taking into account boundary conditions (43) and the symmetry of the problem, one finds that the functions $e n_{\perp x}$ and $e n_{\perp y}$ are expressed by only one constant that coincides with $e n_{\perp y}$. If we denote this constant as $a \cos \theta$ and use

$$
n_{\perp x} \approx \frac{\cos \varphi}{D}, \quad n_{\perp y} \approx \frac{\sin \varphi \cos ^{2} \theta}{D},
$$

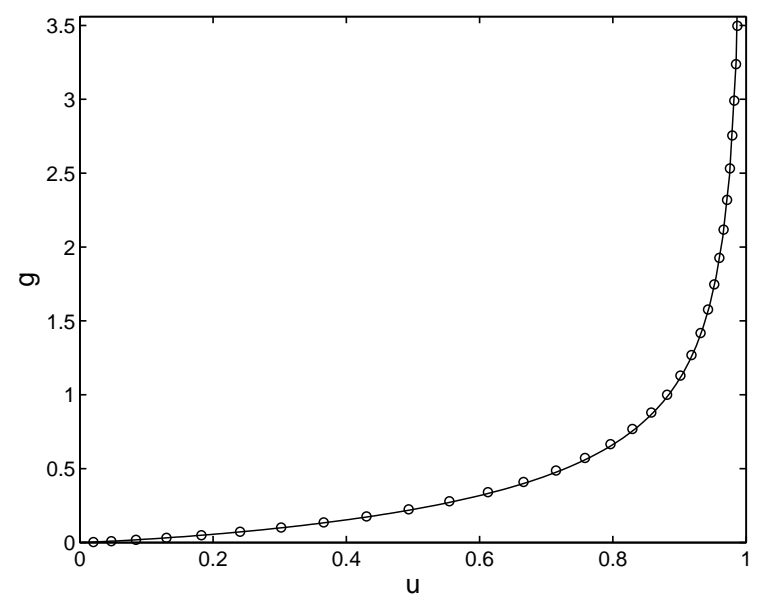

FIG. 6: The function $g(u)$ defined by $u=g \operatorname{arcsinh}(1 / g)$ (circles). Also shown is approximation (47) (solid line).

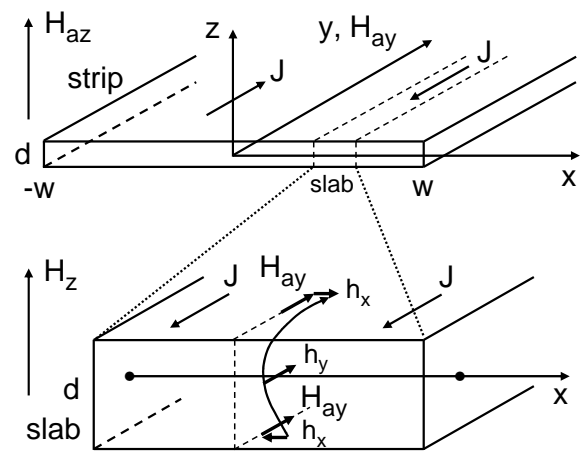

FIG. 7: Schematic picture of the strip. Shown is also a "slab" cut-out near the point $x$, see Sec. III C.

we arrive at formulas (45). The profiles $h_{x}(z)$ and $h_{y}(z)$ follow from Eqs. (26) and (27), and the constant $a$ can be found from the condition

$$
J=J_{y}=\int_{-d / 2}^{d / 2} \frac{d z j_{c \perp} \sin \varphi}{D}
$$

which is just Eq. (46).

It is also instructive to write the electric-field components $E_{x} \equiv e n_{\perp x}$ and $E_{y} \equiv e n_{\perp y}$ explicitly. Using Eqs. (45) and (50), we find

$$
\begin{aligned}
& E_{x}=-\mu_{0} \dot{H}_{a y} z \\
& E_{y}=\mu_{0} \dot{H}_{a y} a \cos \theta=\frac{\mu_{0} \dot{H}_{a y} d}{2} \cos \theta g\left(J \cos \theta / J_{c}\right) .
\end{aligned}
$$

The field $E_{x}$ results from the tilt of a vortex line along the $y$ direction when $H_{a y}$ is applied to the slab. Note that $\int_{-d / 2}^{d / 2} E_{x}(z) d z=0$ since the upper $(z>0)$ and lower $(z<$ $0)$ parts of the vortex move in opposite directions when the tilt occurs. On the other hand, $E_{x}$ is independent of $z$. This component of the electric field is due to a drift of the vortex as a whole in the $x$ direction when $H_{a y}$ is applied to the sample $\underline{\underline{41}}$

The above formulas for the slab with a current enable one to reproduce a number of results for the vortex-shaking effect that were derived from geometrical considerations $\stackrel{40,41}{=}$ In particular, the expression for $\varphi(z)$ in Eqs. (45), formula (46), and Eq. (53), in fact, coincide with Eqs. (4), (6) and (28) from Ref. 41 in which the socalled longitudinal vortex-shaking effect in a thin strip was considered. To obtain the formulas for the vortexshaking effect in a rectangular platelet, $\stackrel{40}{\longleftarrow}$ one should consider the slab with $H_{a z} \gg H_{a y}, J_{c}$ and with the total current $J$ flowing at an arbitrary angle to the $y$ axis, i.e., when $\mathbf{J}=\left(J_{x}, J_{y}\right)$. The appropriate solution of the critical state equations is still obtained from Eqs. (49), but now there is no more symmetry restriction on the $z$ dependences of all the functions, and $e n_{\perp x}$ and $e n_{\perp y}$ are expressed via two constants. Similarly to Eq. (51), these constants can be expressed via $J_{x}$ and $J_{y}$, and the solution thus obtained reproduces the appropriate results of Ref. 40. 


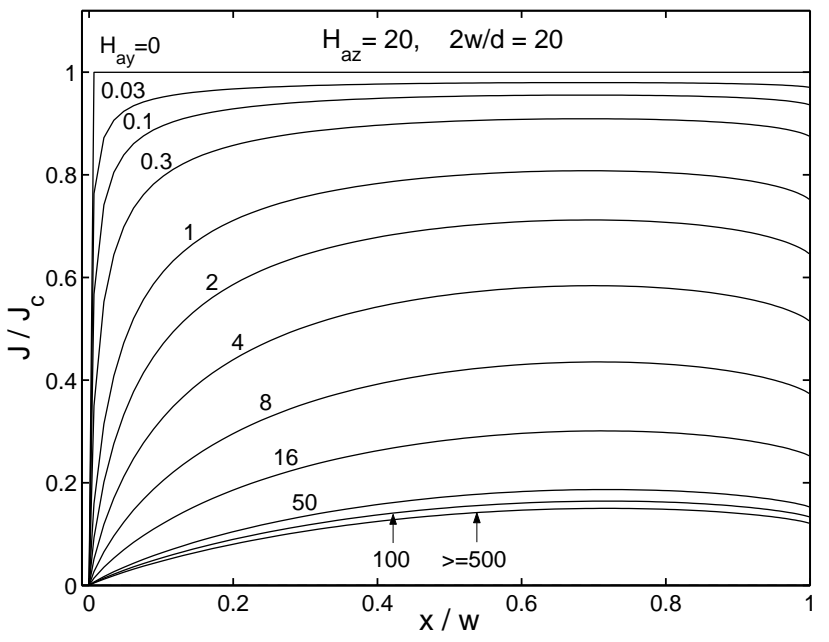

FIG. 8: The sheet current $J(x) \equiv\left|J_{y}(x)\right|$ in the strip to which first a large perpendicular magnetic field $H_{a z}=20$ is applied and then an increasing longitudinal field $H_{a y}$. The aspect ratio of the strip is $2 w / d=20$. The magnetic fields are in units of $J_{c}=j_{c \perp} d$.

\section{Third example: strip}

We now consider the third example of the general $\mathrm{T}$ critical state. Let a thin strip fill the space $|x| \leq w$, $|y|<\infty,|z| \leq d / 2(d \ll w)$, and be in a constant and uniform external magnetic field $H_{a z}$ directed along the $z$ axis, i.e., perpendicularly to the strip plane. The critical current density $j_{c \perp}$ is still assumed to be constant, and let $H_{a z}$ considerably exceed $J_{c}=j_{c \perp} d$ so that at the initial moment of time, $t=0$, the strip is in the fully penetrated Bean critical state. In other words, the magnetic-field profile $H_{z}(x)$ in the strip is described by the well-known function,,$\frac{5,6,7}{=}$ and one has $J_{y}(x)=J_{c}$ for $-w \leq x<0$ and $J_{y}(x)=-J_{c}$ for $w \geq x>0$, where the sheet current $J_{y}$ is the current density integrated over the thickness $d$. At $t>0$ the magnetic field $H_{a y}$ is switched on in the $y$ direction, and hence the applied field is tilted towards the axis of the strip. Note that the critical states in isotropic and anisotropic strips placed in inclined magnetic fields were studied in Refs. 12, 13, 15, 16. However, in all these papers the external magnetic field was tilted perpendicularly to the axis of the strip, the currents in the critical states were always perpendicular to local magnetic fields, and thus, the usual Bean critical states occurred in the strips. In the considered case the general T-critical states develop in the strip, and these states differ from the states of the second example in that the magnetic field $H_{z}$ and the currents $J$ are not uniform in the $x$ direction any more.

Strictly speaking, the description of the magnetic-field tilt towards the axis of the strip reduces to solving a two-dimensional general T-critical state problem. However, the smallness of the parameter $d / w$ enables us to simplify this problem by application of the approach of Ref. 39. Within this approach, we split the problem into

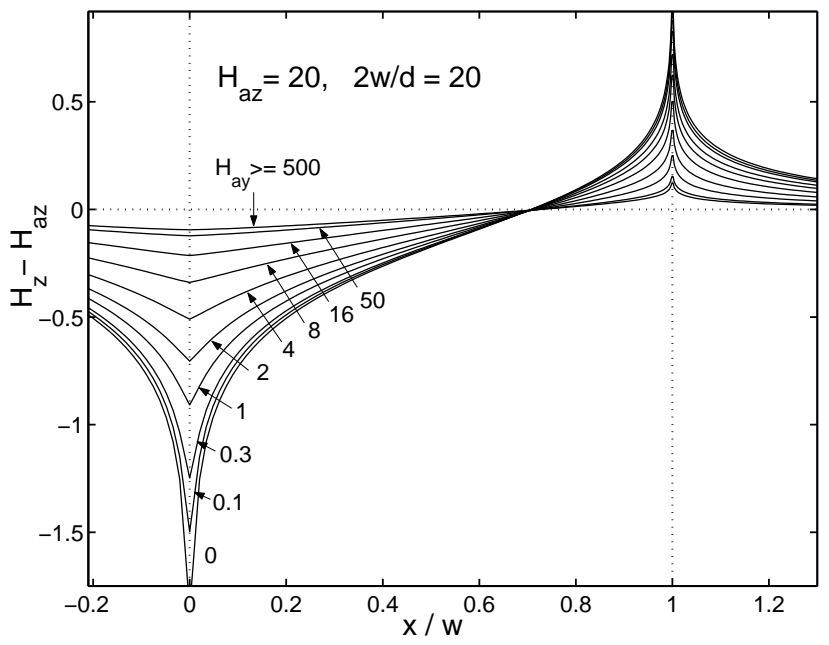

FIG. 9: The perpendicular magnetic field $H_{z}(x)$ caused by the sheet current of Fig. 8 The magnetic fields are in units of $J_{c}$.

two simpler problems: A one-dimensional problem across the thickness of the sample, and a problem for the infinitely thin strip. Namely, we first interpret a small section of the strip around an arbitrary point $x$ (see Fig. 7) as an "infinite" slab of thickness $d$ placed in a perpendicular dc magnetic field $H_{z}(x)$ and in a parallel field $H_{a y}$ and carrying a sheet current $J_{y}(x)$. This is just the problem that has been solved in Sec. III B. We then use the resulting electric field $E_{y}$ obtained for the slab, Eq. (53), as the local electric field $E_{y}(x)$ for an infinitely thin strip, to calculate the temporal evolution of the sheet current $J_{y}(x)$ and of the magnetic field $H_{z}(x)$ in this strip by the method of Ref. 63,64. The resulting equation for $J_{y}(x, t)$ can be written in the form: ${ }^{62}$

$$
\frac{\partial J_{y}(x, t)}{\partial t}=\frac{2}{\pi \mu_{0}} \int_{-w}^{w} \frac{d u}{u-x}\left(\frac{w^{2}-u^{2}}{w^{2}-x^{2}}\right)^{1 / 2} \frac{\partial E_{y}\left(J_{y}\right)}{\partial u},
$$

where $E_{y}\left(J_{y}\right)$ is given by Eq. (53). On determining $J_{y}(x, t)$, the magnetic-field profiles are found from the Biot-Savart law. Since $E_{y}\left(J_{y}\right) \propto \dot{H}_{a y}$, we see again that the temporal dependence of the current and magnetic-field profiles is only a parameterization of their dependence on $H_{a y}$, Sec. II A. It also follows from Eqs. (53) and (54) that these profiles depend on the parameters $H_{a y}, H_{a z}, d, w$ via the following combinations: $J_{y}=J_{y}\left(x / w, H_{a y} / H_{a z}, P\right), H_{z}=$ $H_{z}\left(x / w, H_{a y} / H_{a z}, P\right)$ where we have introduced the notation $P \equiv(d / 2 w) H_{a z} / J_{c}$. Note that the considered critical state problem is similar to the problem of the longitudinal vortex-shaking effect in a thin strip. $\underline{\underline{41}}$ The difference between the problems is that the magnetic field $H_{a y}$ now increases monotonically rather than oscillates about $H_{a y}=0$, and here we present results up to large values of $H_{a y}$ even as compared with $H_{a z}$.

In Figs. 8 and 9 we show the profiles $J\left(x, H_{a y}\right) \equiv$ $\left|J_{y}\left(x, H_{a y}\right)\right|$ and $H_{z}\left(x, H_{a y}\right)$ that develop in the strip 
during increase of the longitudinal field component $H_{a y}$, i.e., when the applied field is tilted away from the $z$ axis towards the strip axis $y$. The profiles $J\left(x, H_{a y}\right)$ take the shape similar to the shape of the profiles in the longitudinal vortex-shaking effect $\frac{41}{1}$, and their magnitude decreases with increasing $H_{a y}$. However, in contrast to the shaking effect, this magnitude does not decrease down to zero but tends to a finite limit that depends on the only parameter $P=(d / 2 w)\left(H_{a z} / J_{c}\right)$. Thus, at $H_{a y} \gg H_{a z}$ the current profiles $J\left(x, H_{a y}\right)$ and the magnetic-field profiles $H_{z}\left(x, H_{a y}\right)$ reach nonzero limiting distributions. The existence of such limiting $J(x)$ and $H_{z}(x)$ can be understood from the following considerations: At small $\cos \theta$, if one neglects logarithmic corrections, the electric field $E_{y}$, Eq. (53), is proportional to $\dot{H}_{a y}(d / 2 w)\left(J / J_{c}\right) \cos ^{2} \theta$, and equation (54) has a solution with separable variables: $J_{y}\left(x, H_{a y}\right)=$ $J_{c} f(x / w) F\left(H_{a y}\right)$ where $f(x / w)$ and $F\left(H_{a y}\right)$ are some functions and $\cos ^{2} \theta=H_{a z}^{2} /\left(H_{a z}^{2}+H_{a y}^{2}\right)$. Inserting this form of $J_{y}\left(x, H_{a y}\right)$ into Eq. (54), we find that

$$
\ln F\left(H_{a y}\right) \propto-P \arctan \frac{H_{a y}}{H_{a z}}+\text { const. }
$$

i.e., at $H_{a y} \rightarrow \infty$ the function $F\left(H_{a y}\right)$ does not tend to zero. In other words, with increasing $H_{a y}$ the decay rate of $J$ decreases so quickly that $J$ does not reach zero even in the limit $H_{a y} \rightarrow \infty$.

In Fig. 10 we show the magnetic moment per unit length of the strip, $M_{z}$, in the general T-critical states developed during increase of $H_{a y}$,

$$
M_{z}\left(H_{a y}\right)=-2 \int_{0}^{w} x\left|J_{y}\left(x, H_{a y}\right)\right| d x .
$$

Here $J_{y}\left(x, H_{a y}\right)$ is the solution of Eq. (54), see Fig. 8. At the initial moment of time, when the usual Bean critical state occurs, we have $M_{z}(0)=-J_{c} w^{2}$. The application of $H_{a y}$ leads to the relaxation of $M_{z}$ towards a saturation value $M_{z}(\infty)$. The normalized moment $M_{z}\left(H_{a y}\right) / M_{z}(0)$ depends only on $H_{a y} / H_{a z}$ and the parameter $P$. All the curves of Fig. 10 may be well fitted by stretched exponentials of the form $s+(1-s) \exp \left[-p\left(H_{a y} / H_{a z}\right)^{q}\right]$, with some $s$ and $p$ depending on $P$ and with $q$ close to $2 / 3.65$ However, an expression useful for all $P$ is suggested by Fig. 10 (bottom), namely,

$$
\frac{M_{z}\left(H_{a y}\right)}{M_{z}(0)} \approx \exp \left\{-1.67\left[P \arctan \left(H_{a y} / H_{a z}\right)\right]^{0.65}\right\} \text {. }
$$

This expression, depicted in Fig. 10 (top and bottom) as dots, gives excellent fits to the numerical results for not too small $P \geq 0.5$, but even for smaller $P$ it is qualitatively correct and only slightly underestimates the exact $M_{z}$ at large $H_{a y} / H_{a z}$.

The saturation values $s=M_{z}(\infty) / M_{z}(0)$ are determined by the above-mentioned limiting current profiles and depend on the only parameter $P=(d / 2 w) H_{a z} / J_{c}$. These values obtained numerically are plotted in the inset of Fig. 10 as circles, while the solid line in this inset
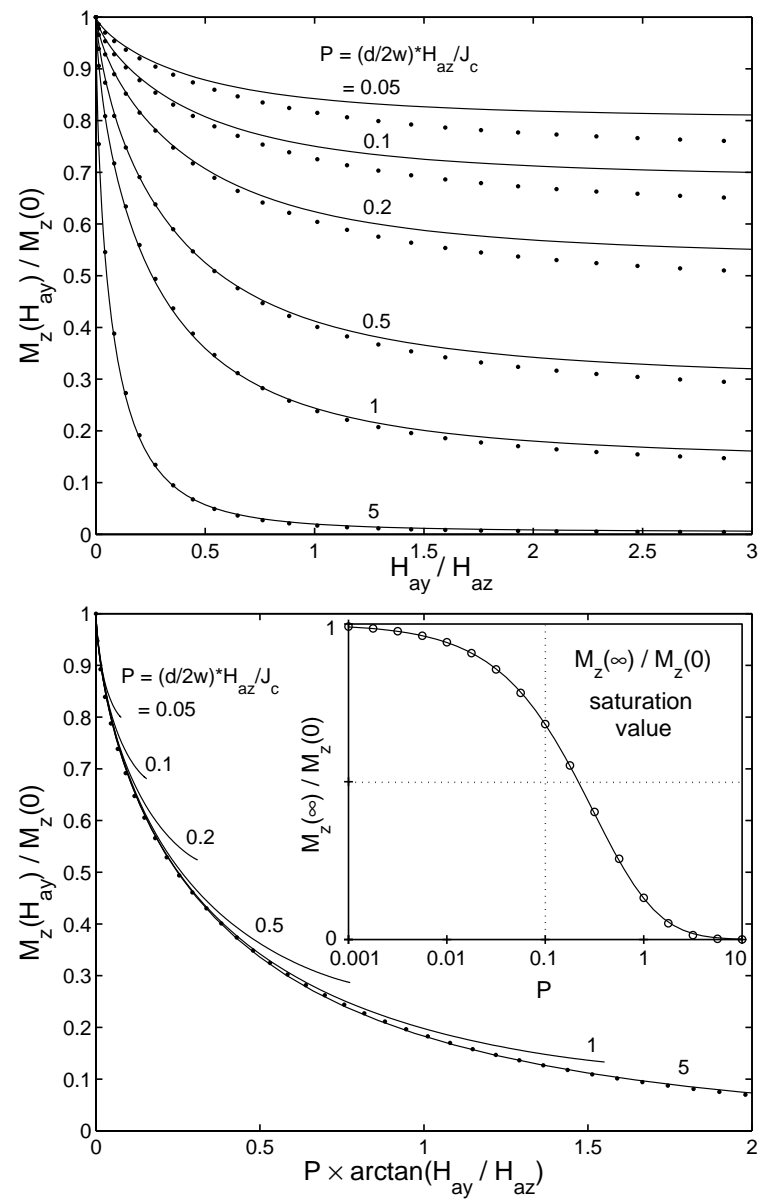

FIG. 10: The perpendicular component $M_{z}$ of the magnetic moment of the strip shown in Fig. 7, plotted versus $H_{a y} / H_{a z}$ (top) and versus $P \cdot \arctan \left(H_{a y} / H_{a z}\right)$ (bottom) for different values of the parameter $P \equiv(d / 2 w) H_{a z} / J_{c}=0.05,0.1,0.2$, $0.5,1,5$. The dots show approximation (57). The inset shows the saturation values $s=M_{z}(\infty) / M_{z}(0)$ calculated numerically (circles) and their fit by Eq. (58) (solid line).

is the following analytic approximation,

$$
s(P) \approx(0.5-0.5 \tanh \{0.41[\ln (P)-0.5]\})^{4} .
$$

When the magnetic field $H_{a y}$ is switched on, not only does the $z$ component $M_{z}$ of the magnetic moment change but there appears also a magnetic moment $M_{y}$ along the axis of the strip. This moment (per unit length of the strip) is defined by the expression:

$$
M_{y}\left(H_{a y}\right)=\int_{-w}^{w} d x \int_{-d / 2}^{d / 2} d z j_{x} z
$$

where the $x$ component of the current density, $j_{x}=$ $j_{c \perp} \cos \varphi / D$, can be found using solution (45). With Eq. (27), formula (59) can be rewritten in the form:

$$
M_{y}\left(H_{a y}\right)=-\int_{-w}^{w} d x \int_{-d / 2}^{d / 2} d z\left[H_{a y}-h_{y}(x, z)\right] .
$$



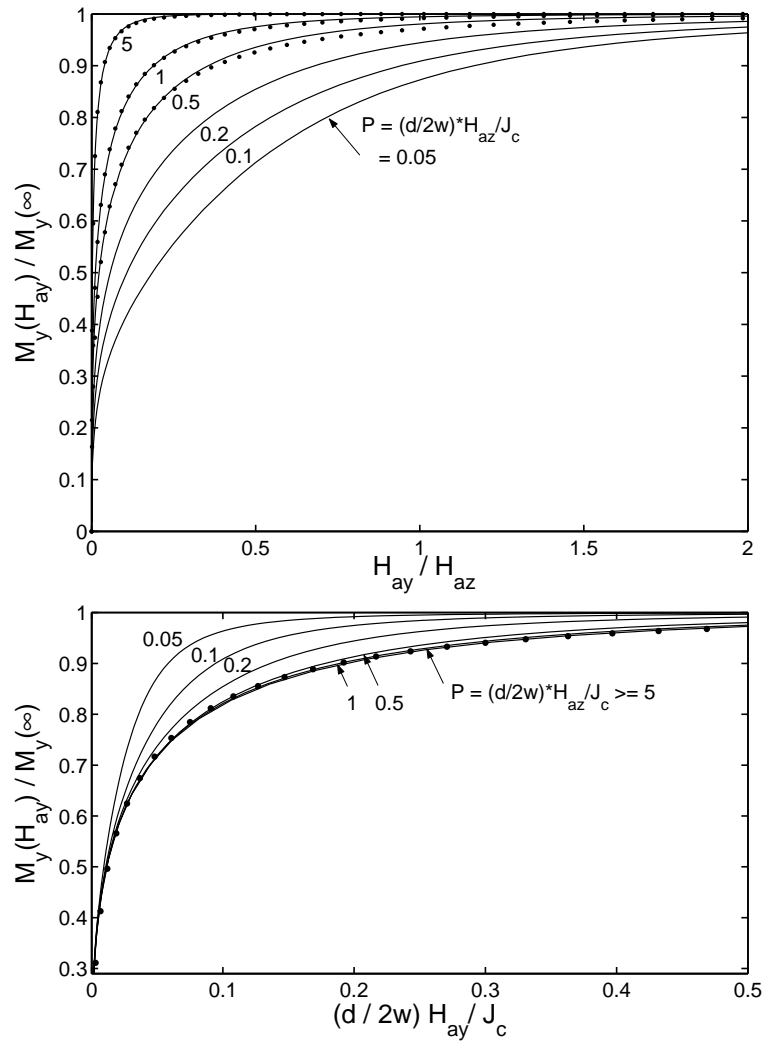

FIG. 11: The longitudinal component $M_{y}$ of the magnetic moment of the strip shown in Fig. 7, plotted versus $H_{a y} / H_{a z}$ (top) and versus $P \cdot H_{a y} / H_{a z}=(d / 2 w) H_{a y} / J_{c}$ (bottom) for the same $P$ as in Fig. 10 . Here $M_{y}(\infty)=M_{y}^{0}=-j_{c \perp} d^{2} w / 2$. Note that the curves for different $P \geq 0.5$ practically coincide if the magnetic moment is plotted versus $P \cdot H_{a y} / H_{a z}$. The dots indicate the fit (62) for this universal curve.

In other words, $M_{y}$ is the "expelled" flux in the $y$ direction. Inserting Eqs. (45) into this formula, we obtain

$$
\frac{M_{y}\left(H_{a y}\right)}{M_{y}^{0}}=\int_{0}^{w} \frac{d x}{w}\left[\sqrt{1+g^{2}(u)}-u g(u)\right]
$$

where $M_{y}^{0}=-j_{c \perp} d^{2} w / 2$ is the magnetic moment in the fully penetrated Bean critical state which occurs if the field $H_{a y}$ alone is applied to the strip, $u=J \cos \theta / J_{c}$, and $J=J\left(x, H_{a y}\right)$ is the current profile obtained from Eq. (54), see Fig. 8.

Figure 11 (top) shows the normalized magnetic moment $M_{y}\left(H_{a y}\right) / M_{a y}(\infty)$ plotted versus $H_{a y} / H_{a z}$ for the same values of the parameter $P$ as in Fig. 10. The saturation value $M_{a y}(\infty)$ always coincides with $M_{y}^{0}=$ $-j_{c \perp} d^{2} w / 2$. Beside this, we find numerically the following interesting result: If $P$ is not too small, $P \geq$ 0.5 , the normalized magnetic moment plotted versus $P \cdot H_{a y} / H_{a z}=(d / 2 w) H_{a y} / J_{c}$ is well described by the unique curve, Fig. 11 (bottom),

$$
\frac{M_{y}\left(H_{a y}\right)}{M_{y}(\infty)} \approx 1-\exp \left\{-4.8\left[(d / 2 w)\left(H_{a y} / J_{c}\right)\right]^{0.44}\right\} \text {. }
$$

At smaller $P$ values fits of the form (62) are still possible, but with different fitting parameters.

The results of this section describe the relaxation of $J\left(x, H_{a y}\right)$ and $H_{z}\left(x, H_{a y}\right)$ to the limiting profiles and of $M_{z}\left(H_{a y}\right)$ and $M_{y}\left(H_{a y}\right)$ to their saturation values. According to Figs. 8-11 and Eqs. (57), (62), this relaxation mainly finishes at some $H_{a y}$ proportional to $\min \left(H_{a z}, 2 w J_{c} / d\right)$ [note that $H_{a z} \gg J_{c}=d j_{c \perp}$ for Eq. (53) to be valid and for the full flux penetration to occur in the initial state]. All these results can be verified in experiments similar, e.g., to those of Refs. 66 67. However, we emphasize that in contrast to Refs. 66 67, the magnetic-field component $H_{a z}$ has to be switched on before $H_{a y}$. This guarantees absence of flux-line cutting for not too large $H_{a y}$, see Eq. (34). If similarly to experiments 66,67 the in-plane magnetic field is switched on before $H_{a z}$, completely different critical states will develop.

\section{CONCLUSIONS}

In this paper we point out how to calculate the general T-critical (cutting-free) states in an arbitrarily-shaped type-II superconductor when the applied magnetic field $\mathbf{H}_{a}$ slowly changes in its magnitude and direction. In accordance with the definition of the general T-critical state, it is assumed here that the external magnetic field changes in such a manner that flux cutting does not occur in the sample. Our approach enables one to take into account the anisotropy of flux-line pinning and the dependence of the critical current density perpendicular to a local magnetic field, $j_{c \perp}$, on the longitudinal component of the current density $j_{\|}$. We also show that the variational principle recently proposed ${ }^{44,45,46}$ cannot give the correct description of the general T-critical states for many situations.

We analyze three examples of the general T-critical states, at least two of which may be investigated experimentally. In particular, we study a seemingly simple problem that has not been solved as yet, viz., we consider the critical states in a slab placed in a uniform perpendicular magnetic field $H_{a z}$ and then two components of the in-plane magnetic field, $H_{a x}$ and $H_{a y}$, are applied successively, Sec. III A. We obtain that one of the in-plane components of the magnetic moment, $M_{x}$, becomes positive with increasing $H_{a y}$ for any sign of $M_{x}$ in the initial state (i.e., at $H_{a y}=0$ ). This paramagnetic effect is due to the fact that the currents in the critical states are not perpendicular to the local magnetic fields. This effect is especially evident when $H_{a z}$ is of the order of the selffields of the slab.

In the other example, we analyze the general T-critical states in a long thin strip placed in a perpendicular magnetic field $H_{a z}$ which then tilts towards the axis of the strip $y$, Sec. III C. When $H_{a y}$, the axial component of the applied magnetic field, increases, the magnetic-field and current profiles across the width of the strip tend to 
limiting profiles, and the components of the magnetic moment, $M_{z}$ and $M_{y}$, reach saturation values. The limiting profiles and the saturation value $M_{z}(\infty)$ for $M_{z}\left(H_{a y}\right)$ are determined by the only parameter $P=(d / 2 w) H_{a z} / J_{c}$ where $d$ and $2 w$ are the thickness and the width of the strip, respectively, and $J_{c}=d j_{c \perp}$. If $P$ is not too large, $P<5$, the limiting profiles and $M_{z}(\infty)$ noticeably differ from zero, while at $P \geq 5$ they become very small and practically vanish. The saturation value for $M_{y}$ is always equal to $M_{y}^{0}=-j_{c \perp} d^{2} w / 2$.

\section{Acknowledgments}

This work was supported by the German Israeli Research Grant Agreement (GIF) No G-901-232.7/2005.
1 C. P. Bean, Phys. Rev. Lett. 8, 250 (1962); Rev. Mod. Phys. 36, 31 (1964).

2 A. M. Campbell and J. E. Evetts, Adv. Phys. 21, 199 (1972).

3 E. H. Brandt, Rep. Progr. Phys. 58, 1465 (1995).

4 Y. B. Kim, C. F. Hempstead, and A. R. Strnad, Phys. Rev. 129, 528 (1963).

${ }^{5}$ E. H. Brandt, M. V. Indenbom, and A. Forkl, Europhys. Lett. 22, 735 (1993).

${ }^{6}$ E. H. Brandt and M. V. Indenbom, Phys. Rev. B 48, 12893 (1993).

7 E. Zeldov, J. R. Clem, M. McElfresh, and M. Darwin, Phys. Rev. B 49, 9802 (1994).

8 P. N. Mikheenko and Yu. E. Kuzovlev, Physica C 204, 229 (1993); J. R. Clem and A. Sanchez, Phys. Rev. B 50, 9355 (1994).

9 I. M. Babich and G. P. Mikitik, Phys. Rev. B 54, 6576 (1996)

10 G. P. Mikitik and E. H. Brandt, Phys. Rev. B 62, 6812 (2000).

11 I. M. Babich, G. P. Mikitik, and E. H. Brandt, Phys. Rev. B 74, 224501 (2006).

12 A. A. Zhukov, G. K. Perkins, Yu. V. Bugoslavsky, A. D. Caplin, Phys. Rev. B 56, 2809 (1997).

13 G. P. Mikitik, E. H. Brandt, and M. Indenbom, Phys. Rev. B 70, 014520 (2004).

14 E. H. Brandt and G. P. Mikitik, Phys. Rev. B 72, 024516 (2005).

15 G. P. Mikitik, E. H. Brandt, Phys. Rev. B 72, 064506 (2005).

16 D. G. Gheorghe, M. Menghini, R. J. Wijngaarden, E. H. Brandt, G. P. Mikitik, W. Goldacker, Phys. Rev. B 73, 224512 (2006).

17 C. Y. Pang, A. M. Campbell, and P. G. MacLaren, IEEE Trans. Magn. 17, 134 (1981).

18 L. Prigozhin, J. Comput. Phys. 129, 190 (1996).

19 L. Prigozhin, J. Comput. Phys. 144, 180 (1998).

$20 \mathrm{Ph}$. Vanderbemden, Z. Hong, T. A. Coombs, S. Denis, M. Ausloos, J. Schwartz, I. B. Rutel, N. Hari Babu, D. A. Cardwell, and A. M. Campbell, cond-mat/0703330 (2007).

21 Ph. Vanderbemden, Z. Hong, T. A. Coombs, M. Ausloos, N. Hari Babu, D. A. Cardwell, and A. M. Campbell, cond-mat/0703362 (2007).

22 J. R. Clem, Phys. Rev. B 26, 2463 (1982).

23 J. R. Clem, Phys. Rev. Lett. 38, 1425 (1977)

24 E. H. Brandt, J. Low Temp. Phys. 44, 33 (1981).

25 J. R. Clem, A. Perez-Gonzalez, Phys. Rev. B 30, 5041 (1984).

26 Note that in contrast to some papers we use the term "double critical state" only for the states in which both the transverse and longitudinal critical current densities are equal to their critical values.

27 A. Perez-Gonzalez, J. R. Clem Phys. Rev. B 31, 7048 (1985).

28 R. Boyer and M. A. R. LeBlanc, Solid State Commun. 24, 261 (1977)

${ }^{29}$ R. Boyer, G. Fillion, and M. A. R. LeBlanc, J. Appl. Phys. 51, 1692 (1980).

30 F. Perez-Rodriguez, A. Perez-Gonzalez, J. R. Clem, G. Gandolfini, and M. A. R. LeBlanc, Phys. Rev. B 56 3473 (1997)

31 M. A. R. LeBlanc, D. LeBlanc, A. Golebiowski, and G. Fillion, Phys. Rev. Lett. 66, 3309 (1991).

32 I. F. Voloshin, A. V. Kalinov, S. E. Savel'ev, L. M. Fisher, V. A. Yampol'skii, and F. Perez-Rodriguez, Zh. Exp. Teor. Fiz. 111, 1071 (1997) [JETP 84, 592 (1997)].

33 S. E. Savel'ev, L. M. Fisher, and V. A. Yampol'skii, Zh. Exp. Teor. Fiz. 112, 936 (1997) [JETP 85, 507 (1997)].

34 L. M. Fisher, A. V. Kalinov, S. E. Savel'ev, I. F. Voloshin, V. A. Yampol'skii, M. A. R. LeBlanc, and S. Hirscher, Physica C 278, 169 (1997).

${ }^{35}$ L. M. Fisher, A. V. Kalinov, I. F. Voloshin, I. V. Baltaga, K. V. Il'enko, and V. A. Yampol'skii, Solid State Commun. 97, 833 (1996).

36 L. M. Fisher, K. V. Il'enko, A. V. Kalinov, M. A. R. LeBlanc, F. Perez-Rodriguez, S. E. Savel'ev, I. F. Voloshin, and V. A. Yampol'skii, Phys. Rev. B 61, $15382(2000)$.

37 G. P. Mikitik and E. H. Brandt, Phys. Rev. B 71, 012510 (2005)

38 This definition is a generalization of the definition used in Ref. 25 for the case of a slab.

39 G. P. Mikitik and E. H. Brandt, Phys. Rev. B 62, 6800 (2000).

40 G. P. Mikitik and E. H. Brandt, Phys. Rev. B 69, 134521 (2004).

41 G. P. Mikitik and E. H. Brandt, Phys. Rev. B 67, 104511 (2003).

42 J. Gilchrist, Supercond. Sci. Technol. 7, 849 (1994).

43 J. Gilchrist, J. Phys. D: Appl. Phys. 5, 2252 (1972); Supercond. Sci. Technol. 3, 93 (1990).

44 A. Badía and C. López, Phys. Rev. Lett. 87, 127004 (2001)

45 A. Badía and C. López, Phys. Rev. B 65, 104514 (2002).

46 A. Badía-Majós and C. López, J. Appl. Phys. 95, 8035 (2004).

47 L. D. Landau and E. M. Lifshits, Electrodynamics of Continuous Media, Course in Theoretical Physics Vol. 8 (Pergamon, London, 1959).

48 J. Bardeen and M. J. Stephen, Phys. Rev. 140, A1197 (1965) 
49 When $\mathbf{H}_{a}$ changes, these boundaries may shift in the superconductor. The new positions of the boundaries are found from the continuity of $\mathbf{H}$ in the sample.

50 Ch. Jooss and V. Born, Phys. Rev. B 73, 094508 (2006).

51 P. Lipavský, J. Koláček, K. Morawetz, E. H. Brandt, and T. J. Yang. Bernoulli Potential in Superconductors. How the Electrostatic Field Helps to Understand Superconductivity. Book Series: Lecture Notes in Physics, Springer, Berlin, in print.

52 C. Romero-Salazar and F. Perez-Rodriguez, Appl. Phys. Lett. 83, 5256 (2003).

53 C. Romero-Salazar and F. Perez-Rodriguez, Physica C 404, 317 (2004).

54 Their approach is also correct for the Bean critical states with a fixed direction of the critical currents.

55 The assumption that $\mathbf{E}$ is parallel to $\mathbf{j}$ was first introduced by C.P. Bean, J. Appl. Phys. 41, 2482 (1970), and was used in a number of papers; see, e.g., citations in Ref. 52 .

56 For a slab with lateral dimension $L \gg d$ one still has $j_{z} \approx 0$ almost everywhere in the sample since in a homogeneous external magnetic field the current density $\mathbf{j}$ changes in the $x-y$ plane on the scale of the order of $L$ (except near the edges of the slab).

57 Strictly speaking, we used $j_{c \perp}=$ const. in our paper, $\stackrel{37}{,}$ while in Ref. $45 j_{c \perp}$ was described by the Kim model $\stackrel{4}{=}$ But for the same $H$-dependence of $j_{c \perp}$ the appropriate equations exactly coincide.

58 The factor $1 / 2$ is absent in this formula to account for the contribution to $\mathbf{M}$ from the U-turn of the currents at the ends of a finite slab or strip; see E. H. Brandt, Phys. Rev. B 49, 9024 (1994) and Ref. 13.

59 A.I. Rykov, S. Tajima, and F.V. Kusmartsev, Phys. Rev. B 55, 8557 (1997).

60 A.E. Koshelev and A.I. Larkin, Phys. Rev. B 52, 13559 (1995).

61 S. J. Park and J. S. Kouvel, Phys. Rev. B 48, 13995 (1993); S. J. Park, J. S. Kouvel, H. B. Radousky, and J. Z. Liu, Phys. Rev. B 48, 13998 (1993).

62 E. H. Brandt and G. P. Mikitik, Phys. Rev. Lett. 89, 027002 (2002)

63 E. H. Brandt, Phys. Rev. B 49, 9024 (1994).

64 E. H. Brandt, Phys. Rev. B 64, 024505 (2001).

${ }^{65}$ Interestingly, the temporal relaxation of the magnetic moment in the longitudinal vortex-shaking effect is also approximated by a stretched exponential with $q=0.64 .41$

66 M. V. Indenbom, A. Forkl, B. Ludescher, H. Kronmüller, H.-U. Habermeier, B. Leibold, G. D'Anna, T. W. Li, P. H. Kes, and A. A. Menovsky, Physica C 226, 325 (1994).

67 M. V. Indenbom, C. J. van der Beek, V. Berseth, W. Benoit, G. D'Anna, A. Erb, E. Walker, and R. Flükiger, Nature 385, 702 (1997). 